Sharif University of Technology
Scientia Iranica
Transactions E: Industrial Engineering
hCIENTIA

\title{
Estimation of general parameters under stratified adaptive cluster sampling based on dual use of auxiliary information
}

\author{
F. Younis* and J. Shabbir \\ Department of Statistics Quaid-i-Azam University, Islamabad, Pakistan.
}

Received 21 December 2018; received in revised form 12 May 2019; accepted 2 September 2019

\author{
KEYWORDS \\ Adaptive cluster \\ sampling; \\ Mean; \\ Variance; \\ Difference estimator; \\ Efficiency.
}

\begin{abstract}
Auxiliary information is used mostly in conjunction with study variables to enhance the efficiency of estimators for population mean, total, and variance. Thompson introduced adaptive cluster sampling as an appropriate sampling scheme for rare and clustered populations. This paper presents difference-type and difference-cum-exponentialratio-type estimators utilizing two auxiliary variables for estimating general parameters under stratified adaptive cluster sampling. The proposed estimators utilize auxiliary information in terms of ranks, variances, and means of auxiliary variables in $h$ th stratum. Expressions for bias and mean square error of the proposed estimators are derived using first-order approximation. This numerical study aims to evaluate the performance of the proposed estimators.

(C) 2021 Sharif University of Technology. All rights reserved.
\end{abstract}

\section{Introduction}

Thompson [1] introduced Adaptive Cluster Sampling (ACS) as an efficient sampling scheme under rare, hidden, and clustered population such as drug addicts, endangered species of animals, fisheries, contagious diseases, rare and precious plants, minerals, and natural resources. ACS begins by taking an initial sample using traditional sampling designs, e.g., simple random sampling with or without replacement, systematic and strip ACS, stratified sampling, inverse sampling, ranked set sampling, two-stage sampling, partial systematic sampling, double sampling, sampling via probability proportional to size, and simple Latin square sampling. Then, the sample using information of neighboring units, which satisfies the pre-specified condition, adaptively increases. Recent advances in the area of ACS

*. Corresponding author. Tel.: +92 3015998181

E-mail address: faryal.younis@stat.qau.edu.pk (F. Younis)

doi: $10.24200 /$ sci.2019.52515.2753 include the works of Chutiman et al. [2], Gattone et al. [3], Yasmeen and Thompson [4], Qureshi et al. [5], Bak [6], Younis and Shabbir [7-10].

In case of a sufficient correlation between the study and auxiliary variables, auxiliary information is used to enhance the precision of estimators. Haq et al. [11] and Shabbir [12] propounded that ranks of auxiliary variables could also be used to increase the efficiency of estimators. In this article, differencetype and difference-cum-exponential-ratio-type estimators were presented utilizing two auxiliary variables to estimate general parameters under Stratified Adaptive Cluster Sampling (SACS). Estimators are proposed assuming that population parameters are known for one auxiliary variable (say $z$ ) and unknown for another auxiliary variable (say $x$ ). We adopt the two-phase sampling scheme using ACS as follows:

1. In Phase 1, a large sample of size $n^{\prime}$ is drawn and information on the auxiliary variables $(x$ and $z$ ) is recorded;

2. In Phase 2, a sub sample of size $n$ is drawn 
from phase one $n^{\prime}$ and information on the study variable $(y)$ and the auxiliary variables $(x$ and $z)$ is accessible.

\subsection{Symbols and Notations}

Consider a finite population of $N$ units partitioned into $L$ strata such that:

$$
\sum_{h=1}^{L} N_{h}=N \text {. }
$$

Let $y_{h i}$ and $\left(x_{h i}, z_{h i}\right)$, be the observed values of the study variable $y$ and the auxiliary variables $(x, z)$, respectively, in the $h^{\text {th }}$ stratum. Let $r\left(x_{h i}\right), r\left(z_{h i}\right)$ be the ranks of two auxiliary variables $(x, z)$ in the $h^{\text {th }}$ stratum.

Let $\bar{w}_{y h}, \bar{w}_{x h}, \bar{w}_{z h}, \bar{r}\left(w_{x}\right)_{h}$, and $\bar{r}\left(w_{z}\right)_{h}$ be the sample means corresponding to population means $\bar{Y}_{w h}$, $\bar{X}_{w h}, \bar{Z}_{w h}, \bar{R}\left(w_{x}\right)_{h}$, and $\bar{R}\left(w_{z}\right)_{h}$, respectively, in the $h^{\text {th }}$ stratum. Let $s_{w_{y h}}^{2}, s_{w_{x h}}^{2}$, and $s_{w_{z h}}^{2}$ be the sample variances corresponding to the population variances $S_{w_{y h}}^{2}, S_{w_{x h}}^{2}$, and $S_{w_{z h}}^{2}$, respectively, in the $h^{\text {th }}$ stratum. Also, let $\hat{C}_{w_{y h}}, \hat{C}_{w_{x h}}$, and $\hat{C}_{w_{z h}}$ be the sample coefficients of variation corresponding to population coefficients of variation $C_{w_{y h}}, C_{w_{x h}}$, and $C_{w_{z h}}$, respectively, in the $h^{\text {th }}$ stratum.

The following notations are used:

$$
\begin{aligned}
\bar{R}\left(w_{u}\right) & =\sum_{h=1}^{L} \frac{N_{h}}{N} \bar{R}\left(w_{u}\right)_{h}, \bar{R}\left(w_{u}\right)_{h} \\
& =\frac{1}{N_{h}} \sum_{i=1}^{N_{h}} r\left(w_{u i}\right)_{h}, \bar{r}\left(w_{u}\right)_{h}=\frac{1}{n_{h}} \sum_{i=1}^{n_{h}} r\left(w_{u i}\right)_{h}, \\
S_{r\left(w_{u}\right)_{h}}^{2} & =\frac{1}{N_{h}-1} \sum_{i=1}^{N_{h}}\left\{r\left(w_{u i}\right)_{h}-\bar{R}\left(w_{u}\right)_{h}\right\}^{2}, C_{r\left(w_{u}\right)_{h}} \\
& =\frac{S_{r\left(w_{u}\right)_{h}}}{\bar{R}\left(w_{u}\right)_{h}}, \quad \forall u=x, z, y .
\end{aligned}
$$

Error terms are defined as:

$$
\begin{aligned}
\zeta_{0 h} & =\frac{\bar{w}_{y h}-\bar{Y}_{w h}}{\bar{Y}_{w h}}, & \zeta_{1 h} & =\frac{\bar{w}_{x h}-\bar{X}_{w h}}{\bar{X}_{w h}}, \\
\zeta_{1 h}^{\prime} & =\frac{\bar{w}_{x h}^{\prime}-\bar{X}_{w h}}{\bar{X}_{w h}}, & \zeta_{2 h} & =\frac{\bar{w}_{z h}-\bar{Z}_{w h}}{\bar{Z}_{w h}}, \\
\zeta_{2 h}^{\prime} & =\frac{\bar{w}_{z h}^{\prime}-\bar{Z}_{w h}}{\bar{Z}_{w h}}, & \zeta_{3 h} & =\frac{s_{w_{y h}}^{2}-S_{w_{y h}}^{2}}{S_{w_{y h}}^{2}}, \\
\zeta_{4 h} & =\frac{s_{w_{x h}}^{2}-S_{w_{x h}}^{2}}{S_{w_{x h}}^{2}}, & \zeta_{4 h}^{\prime} & =\frac{s_{w_{x h}}^{2^{\prime}}-S_{w_{x h}}^{2}}{S_{w_{x h}}^{2}}, \\
\zeta_{5 h} & =\frac{s_{w_{z h}}^{2}-S_{w_{z h}}^{2}}{S_{w_{z h}}^{2}}, & \zeta_{5 h}^{\prime} & =\frac{s_{w_{z h}}^{2^{\prime}}-S_{w_{z h}}^{2}}{S_{w_{z h}}^{2}},
\end{aligned}
$$

$$
\begin{aligned}
\zeta_{6 h} & =\frac{\bar{r}\left(w_{x}\right)_{h}-\bar{R}\left(w_{x}\right)_{h}}{\bar{R}\left(w_{x}\right)_{h}}, \quad \zeta_{6 h}^{\prime}=\frac{\bar{r}^{\prime}\left(w_{x}\right)_{h}-\bar{R}\left(w_{x}\right)_{h}}{\bar{R}\left(w_{x}\right)_{h}}, \\
\zeta_{7_{h}} & =\frac{\bar{r}\left(w_{z}\right)_{h}-\bar{R}\left(w_{z}\right)_{h}}{\bar{R}\left(w_{z}\right)_{h}}, \quad \zeta_{7 h}^{\prime}=\frac{\bar{r}^{\prime}\left(w_{z}\right)_{h}-\bar{R}\left(w_{z}\right)_{h}}{\bar{R}\left(w_{z}\right)_{h}},
\end{aligned}
$$

such that:

$$
\begin{aligned}
& E\left(e_{i h}\right)=0 \quad \forall i=0,1, \ldots, 7 \\
& E\left(e_{i h}^{\prime}\right)=0 \quad \forall i=1,2,4,5,6,7 . \\
& E\left(\zeta_{i h}^{2}\right)=\theta_{h} C_{i}^{2}, \quad E\left(\zeta_{i} \zeta_{j}\right)=\theta_{h} C_{i j h}, \\
& \forall i=w_{y h}, w_{x h}, w_{z h}, r\left(w_{x h}\right), r\left(w_{z h}\right),
\end{aligned}
$$

where $\zeta_{w_{y h}}=\zeta_{0 h}, \zeta_{w_{x h}}=\zeta_{1 h}, \zeta_{w_{z h}}=\zeta_{2 h}, \zeta_{r\left(w_{x h}\right)}=$ $\zeta_{6 h}, \zeta_{r\left(w_{x h}\right)}=\zeta_{7_{h}}$. Also, we have:

$$
\begin{aligned}
& E\left(\zeta_{3 h}^{2}\right)=\theta_{h} \lambda_{40000 h}^{*}, \\
& E\left(\zeta_{4 h}^{2}\right)=\theta_{h} \lambda_{04000 h}^{*}, \\
& E\left(\zeta_{5 h}^{2}\right)=\theta_{h} \lambda_{00040 h}^{*}, \\
& E\left(\zeta_{0 h} \zeta_{3 h}\right)=\theta_{h} C_{w_{y h}} \lambda_{30000 h}, \\
& E\left(\zeta_{0 h} \zeta_{4 h}\right)=\theta_{h} C_{w_{y h}} \lambda_{12000 h}, \\
& E\left(\zeta_{0 h} \zeta_{5 h}\right)=\theta_{h} C_{w_{y h}} \lambda_{10020 h}, \\
& E\left(\zeta_{1 h} \zeta_{3 h}\right)=\theta_{h} C_{w_{x h}} \lambda_{21000 h}, \\
& E\left(\zeta_{1 h} \zeta_{4 h}\right)=\theta_{h} C_{w_{x h}} \lambda_{03000 h}, \\
& E\left(\zeta_{1 h} \zeta_{5 h}\right)=\theta_{h} C_{w_{x h}} \lambda_{01020 h}, \\
& E\left(\zeta_{2 h} \zeta_{3 h}\right)=\theta_{h} C_{w_{z h}} \lambda_{20010 h}, \\
& E\left(\zeta_{2 h} \zeta_{4 h}\right)=\theta_{h} C_{w_{z h}} \lambda_{02010 h}, \\
& E\left(\zeta_{2 h} \zeta_{5 h}\right)=\theta_{h} C_{w_{z h}} \lambda_{00030 h}, \\
& E\left(\zeta_{3 h} \zeta_{4 h}\right)=\theta_{h} \lambda_{22000 h}^{*}, \\
& E\left(\zeta_{3 h} \zeta_{5 h}\right)=\theta_{h} \lambda_{20020 h}^{*}, \\
& E\left(\zeta_{3 h} \zeta_{6 h}\right)=\theta_{h} C_{r\left(w_{x h}\right)} \lambda_{20100 h}, \\
& E\left(\zeta_{3 h} \zeta_{7 h}\right)=\theta_{h} C_{r\left(w_{z h}\right)} \lambda_{20001 h}, \\
& E\left(\zeta_{4 h} \zeta_{5 h}\right)=\theta_{h} \lambda_{02020 h}^{*}, \\
& E\left(\zeta_{4 h} \zeta_{6 h}\right)=\theta_{h} C_{r\left(w_{x h}\right)} \lambda_{02100 h}, \\
& E\left(\zeta_{4 h} \zeta_{7 h}\right)=\theta_{h} C_{r\left(w_{z h}\right)} \lambda_{02001 h}, \\
& E\left(\zeta_{5 h} \zeta_{6 h}\right)=\theta_{h} C_{r\left(w_{x h}\right)} \lambda_{00120 h}, \\
& E\left(\zeta_{5 h} \zeta_{7 h}\right)=\theta_{h} C_{r\left(w_{z h}\right)} \lambda_{00021 h},
\end{aligned}
$$


where:

$$
\begin{aligned}
& E\left(\zeta_{i h}^{\prime} \zeta_{j h}^{\prime}\right)=E\left(\zeta_{i h} \zeta_{j h}^{\prime}\right)=E\left(\zeta_{j h} \zeta_{i h}^{\prime}\right)=\theta_{h}^{\prime} E\left(\zeta_{j h} \zeta_{i h}\right), \\
& E\left(\zeta_{i h}^{\prime 2}\right)=E\left(\zeta_{i h} \zeta_{i h}^{\prime}\right)=\theta_{h}^{\prime} E\left(\zeta_{i h}^{2}\right), \forall i, j=1,2,4,5,6,7 . \\
& \theta_{h}=\left(\frac{1}{n_{h}}-\frac{1}{N_{h}}\right) \\
& C_{w_{y} r\left(w_{x}\right) h}=C_{w_{y h}} C_{r\left(w_{x h}\right)} \rho_{w_{y} r_{w_{x}} h}, \\
& C_{w_{x} r\left(w_{z}\right) h}=C_{w_{x h}} C_{r\left(w_{z h}\right)} \rho_{w_{x} r_{w_{z}} h}, \\
& \theta_{h}^{\prime}=\left(\frac{1}{n_{h}^{\prime}}-\frac{1}{N_{h}}\right) \\
& C_{w_{y} r\left(w_{z}\right) h}=C_{w_{y h}} C_{r\left(w_{z h}\right)} \rho_{w_{y} r_{w_{z}} h}, \\
& C_{w_{x} r\left(w_{x}\right) h}=C_{w_{x h}} C_{r\left(w_{x h}\right)} \rho_{w_{x} r_{w_{x}} h}, \\
& \theta_{h}^{\prime \prime}=\theta_{h}-\theta_{h}^{\prime}, \\
& C_{w_{y} w_{x} h}=C_{w_{y h}} C_{w_{x h}} \rho_{w_{y} w_{x} h} \\
& C_{w_{x} w_{z} h}=C_{w_{x h}} C_{w_{z h}} \rho_{w_{x} w_{z} h} \\
& \lambda_{r s t l v_{h}}=\frac{\mu_{r s t l v_{h}}}{\mu_{20000_{h}}^{\frac{r}{2}} \mu_{02000_{h}}^{\frac{s}{2}} \mu_{00200_{h}}^{\frac{t}{2}} \mu_{00020_{h}}^{\frac{l}{2}} \mu_{00002_{h}}^{\frac{v}{2}}}, \\
& C_{r\left(w_{x}\right) r\left(w_{z}\right) h}=C_{r\left(w_{x h}\right)} C_{r\left(w_{z h}\right)} \rho_{r_{w_{x}} r_{w_{z}} h}, \\
& \lambda_{r_{s t l v_{h}}^{*}}^{*}=\lambda_{r_{s t l v_{h}}}-1, \\
& C_{w_{z} r\left(w_{z}\right) h}=C_{w_{z h}} C_{r\left(w_{z h}\right)} \rho_{w_{z} r_{w_{z}} h}, \\
& C_{w_{z} r\left(w_{x}\right) h}=C_{w_{z h}} C_{r\left(w_{x h}\right)} \rho_{w_{z} r_{w_{x}} h}, \\
& \mu_{r s t l v_{h}}=\frac{1}{N_{h}-1} \sum_{i=1}^{N_{h}} \\
& \left\{\begin{array}{c}
\left(w_{y h_{i}}-\bar{Y}_{w h}\right)^{r}\left(w_{x h_{i}}-\bar{X}_{w h}\right)^{s}\left(r\left(w_{x i}\right)_{h}-\bar{R}\left(w_{x}\right)_{h}\right)^{t} \\
\left(w_{z h_{i}}-\bar{Z}_{w_{h}}\right)^{l}\left(r\left(w_{z i}\right)_{h}-\bar{R}\left(w_{z}\right)_{h}\right)^{v}
\end{array}\right\} .
\end{aligned}
$$

\section{Existing estimators}

\subsection{Estimators for population mean}

Some of the existing estimators for population mean under Simple Random Sampling (SRS) using two auxiliary variables are discussed in this section under SACS.

1. Usual sample mean in SACS is given by:

$$
t_{S-1 m}=\frac{1}{N} \sum_{h=1}^{L} N_{h} \bar{w}_{y h} .
$$

The Mean Square Error (MSE) of $t_{S-1 m}$ to the firstorder approximation is given by:

$$
M S E\left(t_{S-1 m}\right) \cong \frac{1}{N^{2}} \sum_{h=1}^{L} N_{h}^{2} \theta_{h} \bar{Y}_{w h}^{2} C_{w_{y h}}^{2} .
$$

2. Usual ratio estimator for population mean in SACS is given by:

$$
t_{S-2 m}=\frac{1}{N} \sum_{h=1}^{L} N_{h} \bar{w}_{y h}\left(\frac{\bar{w}_{x h}^{\prime}}{\bar{w}_{x h}}\right)\left(\frac{\bar{Z}_{w h}}{\bar{w}_{z h}^{\prime}}\right) .
$$

The bias and MSE of $t_{S-2 m}$ to first-order approximation are given by:

$$
\begin{aligned}
\operatorname{Bias}\left(t_{S-2 m}\right) & \cong \frac{1}{N} \sum_{h=1}^{L} N_{h} \bar{Y}_{w h} \\
& {\left[\theta_{h}^{\prime \prime}\left(C_{w_{x h}}^{2}-C_{w_{y} w_{x} h}\right)+\theta_{h}^{\prime}\left(C_{w_{z h}}^{2}-C_{w_{y} w_{z} h}\right)\right], }
\end{aligned}
$$

and:

$$
\begin{aligned}
M S E\left(t_{S-2 m}\right) \cong & \frac{1}{N^{2}} \sum_{h=1}^{L} N_{h}^{2} \bar{Y}_{w h}^{2} \\
& {\left[\begin{array}{c}
\theta_{h} C_{w_{y h}}^{2}+\theta^{\prime \prime}\left(C_{w_{x h}}^{2}-2 C_{w_{y} w_{x} h}\right) \\
+\theta_{h}^{\prime}\left(C_{w_{z h}}^{2}-2 C_{w_{y} w_{z} h}\right)
\end{array}\right] . }
\end{aligned}
$$

3. Traditional exponential ratio-type estimator for population mean in SACS is given by:

$$
\begin{aligned}
t_{S-3 m}= & \frac{1}{N} \sum_{h=1}^{L} N_{h} \bar{w}_{y h} \exp \left(\frac{\bar{w}_{x h}^{\prime}-\bar{w}_{x h}}{\bar{w}_{x h}^{\prime}+\bar{w}_{x h}}\right) \\
& \exp \left(\frac{\bar{Z}_{w h}-\bar{w}_{z h}^{\prime}}{\bar{Z}_{w h}+\bar{w}_{z h}^{\prime}}\right) .
\end{aligned}
$$

The bias and MSE of $t_{S-3 m}$ to first-order approximation are given by:

$$
\begin{aligned}
\operatorname{Bias}\left(t_{S-3 m}\right) & \cong \frac{1}{N} \sum_{h=1}^{L} N_{h} \bar{Y}_{w h} \\
& {\left[\theta_{h}^{\prime \prime}\left(\frac{3 C_{w_{x h}}^{2}}{8}-\frac{C_{w_{y} w_{x} h}}{2}\right)\right.} \\
& \left.+\theta_{h}^{\prime}\left(\frac{3 C_{w_{z h}}^{2}}{8}-\frac{C_{w_{y} w_{z} h}}{2}\right)\right],
\end{aligned}
$$

and:

$$
\begin{gathered}
M S E\left(t_{S-3 m}\right) \cong \frac{1}{N^{2}} \sum_{h=1}^{L} N_{h}^{2} \bar{Y}_{w h}^{2} \\
{\left[\begin{array}{c}
\theta_{h} C_{w_{y h}}^{2}+\theta_{h}^{\prime \prime}\left(\frac{C_{w_{x h}}^{2}}{4}-C_{w_{y} w_{x} h}\right) \\
+\theta_{h}^{\prime}\left(\frac{C_{w_{z h}}^{2}}{4}-C_{w_{y} w_{z} h}\right)
\end{array}\right]}
\end{gathered}
$$

4. Traditional difference-type estimator for population mean in SACS is given by: 


$$
\begin{aligned}
t_{S-4 m}= & \frac{1}{N} \sum_{h=1}^{L} N_{h}\left[\bar{w}_{y h}+k_{1 h}\left(\bar{w}_{x h}^{\prime}-\bar{w}_{x h}\right)\right. \\
& \left.+k_{2 h}\left(\bar{Z}_{w h}-\bar{w}_{z h}^{\prime}\right)\right],
\end{aligned}
$$

where $k_{1 h}$ and $k_{2 h}$ are constants. The MSE of $t_{S-4 m}$ to first-order approximation is given by:

$$
\begin{gathered}
M S E\left(t_{S-4 m}\right)_{\min } \cong \frac{1}{N^{2}} \sum_{h=1}^{L} N_{h}^{2} \bar{Y}_{w h}^{2} C_{w_{y h}}^{2} \\
\left(\theta_{h}-\theta_{h}^{\prime \prime} \rho_{w_{y} w_{x} h}^{2}-\theta_{h}^{\prime} \rho_{w_{y} w_{z} h}^{2}\right),
\end{gathered}
$$

where:

$$
\begin{aligned}
k_{1 h_{o p t}} & =\frac{\bar{Y}_{w h} C_{w_{y h}} \rho_{w_{y} w_{x} h}}{\bar{X}_{w h} C_{w_{x h}}}, \\
k_{2 h_{o p t}} & =\frac{\bar{Y}_{w h} C_{w_{y h}} \rho_{w_{y} w_{z} h}}{\bar{Z}_{w h} C_{w_{z h}}} .
\end{aligned}
$$

5. Based on Shabbir and Gupta [13], the exponential ratio-type estimator for population mean in SACS is given by:

$$
\begin{aligned}
t_{S-5 m} & =\frac{1}{N} \sum_{h=1}^{L} N_{h} \bar{w}_{y h} \exp \left(\frac{\xi_{3 h}\left(\bar{w}_{x h}^{\prime}-\bar{w}_{x h}\right)}{\bar{w}_{x h}^{\prime}+\left(k_{3 h}-1\right) \bar{w}_{x h}}\right) \\
& \exp \left(\frac{\xi_{4 h}\left(\bar{w}_{z h}^{\prime}-\bar{w}_{z h}\right)}{\bar{w}_{z h}^{\prime}+\left(k_{4 h}-1\right) \bar{w}_{z h}}\right) \\
& \exp \left(\frac{\xi_{5 h}\left(\bar{w}_{z h}^{\prime}-\bar{Z}_{w h}\right)}{\bar{w}_{z h}^{\prime}+\left(k_{5 h}-1\right) \bar{Z}_{w h}}\right),
\end{aligned}
$$

where $\left(\xi_{3 h}, \xi_{4 h}, \xi_{5 h}\right)$ and $\left(k_{3 h}, k_{4 h}, k_{5 h}\right)$ are constants. The bias and minimum MSE of $t_{S-5 m}$ at optimum values of constants to first-order approximation are given by:

$$
\begin{array}{r}
\operatorname{Bias}\left(t_{S-5 m}\right) \cong \frac{1}{N} \sum_{h=1}^{L} N_{h} \bar{Y}_{w h}\left[\theta_{h}^{\prime}\left\{\frac{C_{w_{y} w_{z} h}}{k_{5 h}}-\frac{C_{w_{z h}}^{2}}{2 k_{5 h}^{2}}\right\}\right. \\
+\theta_{h}^{\prime \prime}\left\{\frac{C_{w_{x h}}^{2}}{k_{3 h}}-\frac{C_{w_{y} w_{x} h}}{k_{3 h}}-\frac{C_{w_{x h}}^{2}}{2 k_{3 h}^{2}}\right. \\
\left.\left.+\frac{C_{w_{z h}}^{2}}{k_{4 h}}-\frac{C_{w_{y} w_{z} h}}{k_{4 h}}-\frac{C_{w_{z} h}^{2}}{2 k_{4 h}^{2}}+\frac{C_{w_{x} w_{z} h}}{k_{3 h} k_{4 h}}\right\}\right],
\end{array}
$$

and:

$$
\begin{gathered}
M S E\left(t_{S-5 m}\right)_{\min } \cong \frac{1}{N^{2}} \sum_{h=1}^{L} N_{h}^{2} \bar{Y}_{w h}^{2} C_{w_{y} h}^{2} \\
{\left[\theta_{h}-\theta_{h}^{\prime} \rho_{w_{y} w_{z} h}^{2}-\frac{\theta_{h}^{\prime \prime}}{1-\rho_{w_{x} w_{z} h}^{2}}\left\{\rho_{w_{y} w_{x} h}^{2}\right.\right.}
\end{gathered}
$$

$$
\left.\left.+\rho_{w_{y} w_{z} h}^{2}-2 \rho_{w_{y} w_{x} h} \rho_{w_{y} w_{z} h} \rho_{w_{x} w_{z} h}\right\}\right],
$$

where:

$$
\begin{aligned}
& \left(\frac{\xi}{k}\right)_{3 h_{o p t}}=\frac{C_{w_{y} h}\left(\rho_{w_{y} w_{x} h}-\rho_{w_{y} w_{z} h} \rho_{w_{x} w_{z} h}\right)}{C_{w_{x h}}\left(1-\rho_{w_{x} w_{z} h}^{2}\right)} \\
& \left(\frac{\xi}{k}\right)_{4 h_{o p t}}=\frac{C_{w_{y h}}\left(\rho_{w_{y} w_{z} h}-\rho_{w_{y} w_{x} h} \rho_{w_{x} w_{z} h}\right)}{C_{w_{z h}}\left(1-\rho_{w_{x} w_{z} h}^{2}\right)} \\
& \left(\frac{\xi}{k}\right)_{5 h_{o p t}}=\frac{-C_{w_{y} w_{z} h}}{C_{w_{z h}}^{2}}
\end{aligned}
$$

6. Based on Gupta and Shabbir [14], the ratio-type estimator for population mean in SACS is given by:

$$
t_{S-6 m}=\frac{1}{N} \sum_{h=1}^{L} N_{h} \bar{w}_{y h}\left(\frac{\bar{w}_{x h}^{\prime}}{\bar{w}_{x h}}\right)^{k_{6 h}}\left(\frac{\bar{Z}_{w h}}{\bar{w}_{z h}^{\prime}}\right)^{k_{7 h}}
$$

where $\left(k_{6 h}, k_{7 h}\right)$ are constants. The bias and minimum MSE of $t_{S-6 m}$ at optimum values of constants to first-order approximation are given by:

$$
\begin{aligned}
\operatorname{Bias}\left(t_{S-6 m}\right) \cong \frac{1}{N} \sum_{h=1}^{L} N_{h} \bar{Y}_{w h} \\
{\left[\theta_{h}^{\prime \prime}\left\{\frac{k_{6 h}\left(k_{6 h}+1\right)}{2} C_{w_{x h}}^{2}-k_{6 h} C_{w_{y} w_{x} h}\right\}\right.} \\
\left.+\theta_{h}^{\prime}\left\{\frac{k_{7 h}\left(k_{7 h}+1\right)}{2} C_{w_{z h}}^{2}-k_{7 h} C_{w_{y} w_{z} h}\right\}\right],
\end{aligned}
$$

and:

$$
\begin{gathered}
M S E\left(t_{S-6 m}\right)_{\min } \cong \frac{1}{N^{2}} \sum_{h=1}^{L} N_{h}^{2} \bar{Y}_{w h}^{2} C_{w_{y h}}^{2} \\
{\left[\theta_{h}-\theta_{h}^{\prime \prime} \rho_{w_{y} w_{x} h}^{2}-\theta_{h}^{\prime} \rho_{w_{y} w_{z} h}^{2}\right]}
\end{gathered}
$$

where:

$$
k_{6 h_{o p t}}=\frac{C_{w_{y} w_{x} h}}{C_{w_{x h}}^{2}} \text { and } k_{7 h_{o p t}}=\frac{C_{w_{y} w_{z} h}}{C_{w_{z h}}^{2}} .
$$

7. According to Singh et al. [15], the exponential ratiotype estimator for population mean in SACS is given by:

$$
\begin{aligned}
t_{S-7 m}= & \frac{1}{N} \sum_{h=1}^{L} N_{h} \bar{w}_{y h} \exp \left[k_{8 h}\left(\frac{\bar{w}_{x h}^{\prime}-\bar{w}_{x h}}{\bar{w}_{x h}^{\prime}+\bar{w}_{x h}}\right)\right. \\
& \left.+k_{9 h}\left(\frac{\bar{w}_{z h}^{\prime}-\bar{Z}_{w h}}{\bar{w}_{z h}^{\prime}+\bar{Z}_{w h}}\right)\right]
\end{aligned}
$$

where $\left(k_{8 h}, k_{9 h}\right)$ are constants. The bias and mini- 
mum MSE of $t_{S-7 m}$ at optimum values of constants to first-order approximation are given by:

$$
\begin{aligned}
\operatorname{Bias}\left(t_{S-7 m}\right) \cong \frac{1}{N} \sum_{h=1}^{L} N_{h} \bar{Y}_{w h}\left[\theta _ { h } ^ { \prime \prime } \left\{\frac{k_{8 h}^{2}}{8} C_{w_{x h}}^{2}\right.\right. \\
\left.+\frac{k_{8 h}}{4} C_{w_{x h}}^{2}-\frac{k_{8 h}}{2} C_{w_{y} w_{x} h}\right\} \\
\left.+\theta_{h}^{\prime}\left\{\frac{k_{9 h}^{2}}{8} C_{w_{z h}}^{2}-\frac{k_{9 h}}{4} C_{w_{z h}}^{2}+\frac{k_{9 h}}{2} C_{w_{y} w_{z} h}\right\}\right],
\end{aligned}
$$

and:

$$
\begin{gathered}
M S E\left(t_{S-7 m}\right)_{\min } \cong \frac{1}{N^{2}} \sum_{h=1}^{L} N_{h}^{2} \bar{Y}_{w h}^{2} C_{w_{y} h}^{2} \\
{\left[\theta_{h}-\theta_{h}^{\prime \prime} \rho_{w_{y} w_{x} h}^{2}-\theta_{h}^{\prime} \rho_{w_{y} w_{z} h}^{2}\right]}
\end{gathered}
$$

where:

$$
k_{8 h_{o p t}}=\frac{2 C_{w_{y} w_{x} h}}{C_{w_{x h}}^{2}} \text { and } k_{9 h_{o p t}}=\frac{-2 C_{w_{y} w_{z} h}}{C_{w_{z h}}^{2}} .
$$

8. According to Choudhury and Singh [16], the ratiotype estimator for population mean in SACS is given by:

$$
\begin{aligned}
t_{S-8 m}= & \frac{1}{N} \sum_{h=1}^{L} N_{h} \bar{w}_{y h}\left[k_{10 h}\left(\frac{\bar{w}_{x h}^{\prime}}{\bar{w}_{x h}}\right)\left(\frac{\bar{Z}_{w h}}{\bar{w}_{z h}^{\prime}}\right)\right. \\
& \left.+\left(1-k_{10 h}\right)\left(\frac{\bar{w}_{x h}}{\bar{w}_{x h}^{\prime}}\right)\left(\frac{\bar{w}_{z h}^{\prime}}{\bar{Z}_{w h}}\right)\right],
\end{aligned}
$$

where $k_{10 h}$ is a constant. The bias and minimum MSE of $t_{S-8 m}$ at optimum value of constant to first order of approximation are given by:

$$
\begin{aligned}
\operatorname{Bias}( & \left.t_{S-8 m}\right) \cong \frac{1}{N} \sum_{h=1}^{L} N_{h} \bar{Y}_{w h}\left[\theta _ { h } ^ { \prime \prime } \left\{C_{w_{y} w_{x} h}\right.\right. \\
& \left.+k_{10 h}\left(C_{w_{x h}}^{2}-2 C_{w_{y} w_{x} h}\right)\right\} \\
& \left.+\theta_{h}^{\prime}\left\{C_{w_{y} w_{z} h}+k_{10 h}\left(C_{w_{z h}}^{2}-2 C_{w_{y} w_{z} h}\right)\right\}\right]
\end{aligned}
$$

and:

$$
M S E\left(t_{S-8 m}\right)_{\min } \cong \frac{1}{N^{2}} \sum_{h=1}^{L} N_{h}^{2} \bar{Y}_{w h}^{2}\left(A_{h}-\frac{F_{h}^{2}}{B_{h}}\right)_{(22)}
$$

where:

$$
\begin{aligned}
& A_{h}=\theta_{h} C_{w_{y h}}^{2}+F_{h}, \quad B_{h}=\theta_{h}^{\prime \prime} C_{w_{x h}}^{2}+\theta_{h}^{\prime} C_{w_{z h}}^{2}, \\
& k_{10 h_{o p t}}=\frac{F_{h}}{2 B_{h}},
\end{aligned}
$$

$$
F_{h}=\theta_{h}^{\prime \prime}\left(C_{w_{y} w_{x} h}+C_{w_{x h}}^{2}\right)+\theta_{h}^{\prime}\left(C_{w_{y} w_{z} h}+C_{w_{z h}}^{2}\right) .
$$

9. Hamad et al. [17] presented the difference ratio-type estimator for population mean in SACS below:

$$
\begin{aligned}
t_{S-9 m}= & \frac{1}{N} \sum_{h=1}^{L} N_{h}\left[\bar{w}_{y h}+k_{11 h}\left(\bar{w}_{x h}^{\prime}-\bar{w}_{x h}\right)\right] \\
& {\left[k_{12 h} \frac{\bar{w}_{z h}^{\prime}}{\bar{w}_{z h}}+\left(1-k_{12 h}\right) \frac{\bar{w}_{z h}}{\bar{w}_{z h}^{\prime}}\right], }
\end{aligned}
$$

where $\left(k_{11 h}, k_{12 h}\right)$ are constants. The bias and minimum MSE of $t_{S-9 m}$ at optimum values of constants to first-order approximation are given by:

$$
\begin{aligned}
\operatorname{Bias}( & \left.t_{S-9 m}\right) \cong \frac{1}{N} \sum_{h=1}^{L} N_{h}\left[\bar{Y}_{w h} C_{w_{y} w_{z} h} \theta_{h}^{\prime \prime}\right. \\
& +\bar{Y}_{w h} k_{12 h} \theta_{h}^{\prime \prime}\left(C_{w_{z h}}^{2}-2 C_{w_{y} w_{z} h}\right) \\
& -\bar{X}_{w h} k_{11 h} \theta_{h}^{\prime \prime} C_{w_{x} w_{z} h}+2 k_{11 h} k_{12 h} \bar{X}_{w h} \theta_{h}^{\prime \prime} \\
& \left.C_{w_{x} w_{z} h}\right]
\end{aligned}
$$

and:

$$
\begin{gathered}
M S E\left(t_{S-9 m}\right)_{\min } \cong \frac{1}{N^{2}} \sum_{h=1}^{L} N_{h}^{2} \bar{Y}_{w h}^{2} \\
\left(A_{h}+\frac{F_{h} G_{h} H_{h}-D_{h} G_{h}^{2}-B_{h} H_{h}^{2}}{4 B_{h} D_{h}-F_{h}^{2}}\right),
\end{gathered}
$$

where:

$$
\begin{aligned}
& A_{h}=\theta_{h} C_{w_{y h}}^{2}+\theta_{h}^{\prime \prime}\left(2 C_{w_{y} w_{z} h}+C_{w_{z h}}^{2}\right), \\
& B_{h}=4 \theta_{h}^{\prime \prime} C_{w_{z h}}^{2}, \quad F_{h}=4 \theta_{h}^{\prime \prime} C_{w_{x} w_{z} h}, \\
& D_{h}=\theta_{h}^{\prime \prime} C_{w_{x h}}^{2}, \quad G_{h}=4 \theta_{h}^{\prime \prime}\left(C_{w_{y} w_{z} h}-C_{w_{z h}}^{2}\right), \\
& k_{11 h_{o p t}}=\frac{\bar{Y}_{w_{h}}\left(2 H_{h} B_{h}-F_{h} G_{h}\right)}{\bar{X}_{w h}\left(4 B_{h} D_{h}-F_{h}^{2}\right)}, \\
& H_{h}=2 \theta_{h}^{\prime \prime}\left(C_{w_{y} w_{x} h}-C_{w_{x} w_{z} h}\right), \\
& k_{12 h_{o p t}}=\frac{2 G_{h} D_{h}-F_{h} H_{h}}{4 B_{h} D_{h}-F_{h}^{2}} .
\end{aligned}
$$

10. Chutiman [18], Yadav et al. [19], and Qureshi et al. [20] proposed the ratio-type estimator for population mean in SACS as follows:

$$
\begin{aligned}
t_{S-10 m_{j}}= & \frac{1}{N} \sum_{h=1}^{L} N_{h} \bar{w}_{y h}\left(\frac{k_{13 h_{x j}} \bar{w}_{x h}^{\prime}+k_{14 h_{x j}}}{k_{13 h_{x j}} \bar{w}_{x h}+k_{14 h_{x j}}}\right) \\
& \left(\frac{k_{13 h_{z j}} \bar{Z}_{w h}+k_{14 h_{z j}}}{k_{13 h_{z j}} \bar{w}_{z h}^{\prime}+k_{14 h_{z j}}}\right)
\end{aligned}
$$


Table 1. Combinations of constants for $t_{S-10 m_{j}}$.

\begin{tabular}{ccccc}
\hline$j$ & $k_{13 h_{x j}}$ & $k_{14 h_{x j}}$ & $k_{13 h_{z j}}$ & $k_{14 h_{z j}}$ \\
\hline \multicolumn{5}{c}{$[18]$} \\
\hline 1 & 1 & $C_{w_{x h}}$ & 1 & $C_{w_{z h}}$ \\
2 & 1 & $\beta_{2\left(w_{x h}\right)}$ & 1 & $\beta_{2\left(w_{z h}\right)}$ \\
3 & $\beta_{2\left(w_{x h}\right)}$ & $C_{w_{x h}}$ & $\beta_{2\left(w_{z h}\right)}$ & $C_{w_{z h}}$ \\
4 & $C_{w_{x h}}$ & $\beta_{2\left(w_{x h}\right)}$ & $C_{w_{z h}}$ & $\beta_{2\left(w_{z h}\right)}$ \\
\hline & \multicolumn{5}{c}{$[19]$} \\
\hline 5 & 1 & $\rho_{w_{y} w_{x} h}$ & $\rho_{w_{y} w_{z h}}$ \\
6 & $\beta_{2\left(w_{x h}\right)}$ & $\beta_{1\left(w_{x h}\right)}$ & $\beta_{2\left(w_{z h}\right)}$ & $\beta_{1\left(w_{z h}\right)}$ \\
7 & $\beta_{1\left(w_{x h}\right)}$ & $\beta_{2\left(w_{x h}\right)}$ & $\beta_{1\left(w_{z h}\right)}$ & $\beta_{2\left(w_{z h}\right)}$ \\
\hline \multirow{5}{*}{} & & {$[20]$} & & \\
\hline 8 & $M R_{\left(w_{x h}\right)}$ & $\beta_{1\left(w_{x h}\right)}$ & $M R_{\left(w_{z h}\right)}$ & $\beta_{1\left(w_{z h}\right)}$ \\
9 & $M R_{\left(w_{x h}\right)}$ & $T M_{\left(w_{x h}\right)}$ & $M R_{\left(w_{z h}\right)}$ & $T M_{\left(w_{z h}\right)}$ \\
10 & $H L_{\left(w_{x h}\right)}$ & $\beta_{1\left(w_{x h}\right)}$ & $H L_{\left(w_{z h}\right)}$ & $\beta_{1\left(w_{z h}\right)}$ \\
11 & $H L_{\left(w_{x h}\right)}$ & $T M_{\left(w_{x h}\right)}$ & $H L_{\left(w_{z h}\right)}$ & $T M_{\left(w_{z h}\right)}$ \\
\hline & & &
\end{tabular}

where $\left(k_{13 h_{x j}}, k_{14 h_{x j}}, k_{13 h_{z j}}, k_{14 h_{z j}}\right)$ are the constants that assume different values for $j=1,2, \ldots$, 11 as given in Table 1.

The bias and MSE of $t_{S-10 m_{j}}$ to first-order approximation are given by:

$$
\begin{gathered}
\operatorname{Bias}\left(t_{S-10 m_{j}}\right) \cong \frac{1}{N} \sum_{h=1}^{L} N_{h} \bar{Y}_{w h} \\
{\left[\begin{array}{c}
\theta_{h}^{\prime \prime}\left\{Q_{x h}^{2} C_{w_{x h}}^{2}-Q_{x h} C_{w_{y} w_{x} h}\right\} \\
+\theta_{h}^{\prime}\left\{Q_{z h}^{2} C_{w_{z h}}^{2}-Q_{z h} C_{w_{y} w_{z} h}\right\}
\end{array}\right],}
\end{gathered}
$$

and:

$$
\begin{aligned}
M S E\left(t_{S-10 m_{j}}\right) & \cong \bar{Y}_{w h}^{2}\left[\theta_{h} C_{w_{y h}}^{2}+\theta_{h}^{\prime \prime}\left\{Q_{x h}^{2} C_{w_{x h}}^{2}\right.\right. \\
& \left.-2 Q_{x h} C_{w_{y} w_{x} h}\right\}+\theta_{h}^{\prime}\left\{Q_{z h}^{2} C_{w_{z h}}^{2}\right. \\
& \left.\left.-2 Q_{z h} C_{w_{y} w_{z} h}\right\}\right]
\end{aligned}
$$

where:

$$
\begin{aligned}
Q_{x h} & =\frac{k_{13 h_{x j}} \bar{X}_{w h}}{k_{13 h_{x j}} \bar{X}_{w h}+k_{14 h_{x j}}}, \\
Q_{z h} & =\frac{k_{13 h_{z j}} \bar{Z}_{w h}}{k_{13 h_{z j}} \bar{Z}_{w h}+k_{14 h_{z j}}} .
\end{aligned}
$$

11. Vishwakarma and Gangele [21] presented the exponential ratio-type estimator for population mean in SACS:

$$
\begin{aligned}
& t_{S-11 m}=\frac{1}{N} \sum_{h=1}^{L} N_{h} \bar{w}_{y h} \exp \\
& \left(\frac{\frac{\bar{w}_{x h}^{\prime}}{k_{15 h} \bar{w}_{z h}^{\prime}+k_{16 h}}\left(k_{15 h} \bar{Z}_{w h}+k_{16 h}\right)-\bar{w}_{x h}}{\frac{\bar{w}_{x h}^{\prime}}{k_{15 h} \bar{w}_{z h}^{\prime}+k_{16 h}}\left(k_{15 h} \bar{Z}_{w h}+k_{16 h}\right)+\bar{w}_{x h}}\right),
\end{aligned}
$$

where $\left(k_{15 h}, k_{16 h}\right)$ are constants. The bias and minimum MSE of $t_{S-11 m}$ at optimum values of constants to first-order approximation are given by:

$$
\begin{aligned}
\operatorname{Bias}( & \left.t_{S-11 m}\right) \cong \frac{1}{N} \sum_{h=1}^{L} N_{h} \bar{Y}_{w h} \\
& {\left[\theta_{h}^{\prime \prime}\left\{\frac{3}{8} Q_{h}^{2} C_{w_{x h}}^{2}-\frac{1}{2} Q_{h} C_{w_{y} w_{x} h}\right\}\right.} \\
& -\theta_{h}^{\prime}\left\{\frac{1}{8} Q_{h}^{2} C_{w_{z h}}^{2}-\frac{1}{2} Q_{h} C_{w_{y} w_{z} h}\right. \\
& \left.\left.+\frac{1}{2} Q_{h} C_{w_{x} w_{z} h}\right\}\right]
\end{aligned}
$$

and:

$$
\begin{gathered}
M S E\left(t_{S-11 m}\right) \cong \frac{1}{N^{2}} \sum_{h=1}^{L} N_{h}^{2} \bar{Y}_{w h}^{2}\left[\theta_{h} C_{w_{y h}}^{2}\right. \\
\left.-\theta_{h}^{\prime} C_{w_{y} h}^{2} \rho_{w_{y} w_{z} h}^{2}+\theta_{h}^{\prime \prime}\left(\frac{C_{w_{x h}}^{2}}{4}-C_{w_{y} w_{x} h}\right)\right]
\end{gathered}
$$

where:

$$
\begin{aligned}
& Q_{h}=\frac{k_{15 h} \bar{Z}_{w h}}{k_{15 h} \bar{Z}_{w h}+k_{16 h}}, \\
& Q_{h_{o p t}}=\frac{2 C_{w_{y h}} \rho_{w_{y} w_{z} h}}{C_{w_{z h}}} .
\end{aligned}
$$

12. Singh and Khalid [22] gave the exponential ratiotype estimator for population mean in SACS below:

$$
t_{S-12 m}=\frac{1}{N} \sum_{h=1}^{L} N_{h} \bar{w}_{y h} \exp \left(\frac{\bar{w}_{x h}^{\prime} \bar{Z}_{w h}^{*}-\bar{w}_{x h}}{\frac{\bar{z}_{x h}^{\prime}}{\bar{Z}_{w h}} \bar{Z}_{w h}^{*}+\bar{w}_{x h}}\right),
$$

where $\bar{Z}_{w h}^{*}=\frac{N_{h} \bar{Z}_{w h}-n_{h}^{\prime} \bar{w}_{z h}^{\prime}}{N_{h}-n_{h}^{\prime}}$. The bias and MSE of $t_{S-12 m}$ to first-order approximation are given by:

$$
\begin{gathered}
\operatorname{Bias}\left(t_{S-12 m}\right) \cong \frac{1}{N} \sum_{h=1}^{L} N_{h} \bar{Y}_{w h} \\
{\left[\theta_{h}^{\prime \prime}\left\{\frac{3}{8} C_{w_{x h}}^{2}+\frac{1}{2} C_{w_{y} w_{x} h}\right\}\right.} \\
\left.+\theta_{h}^{\prime}\left\{\frac{a_{h}^{2}}{8} C_{w_{z h}}^{2}+\frac{a_{h}}{2} C_{w_{y} w_{z} h}\right\}\right],
\end{gathered}
$$

and: 


$$
\begin{gathered}
M S E\left(t_{S-12 m}\right) \cong \frac{1}{N^{2}} \sum_{h=1}^{L} N_{h}^{2} \bar{Y}_{w h}^{2} \\
{\left[\theta_{h} C_{w_{y h}}^{2}+\theta_{h}^{\prime \prime}\left(\frac{C_{w_{x h}}^{2}}{4}-C_{w_{y} w_{x} h}\right)\right.} \\
\left.+\theta_{h}^{\prime} a_{h}\left(\frac{C_{w_{z h}}^{2}}{4}-C_{w_{y} w_{x} h}\right)\right],
\end{gathered}
$$

where $a_{h}=\frac{n_{h}^{\prime}}{N_{h}-n_{h}^{\prime}}$.

13. Khan and Al-Hossain [23] proposed a differencetype estimator for population mean in SACS:

$$
\begin{aligned}
t_{S-13 m}= & \frac{1}{N} \sum_{h=1}^{L} N_{h}\left[\bar{w}_{y h}+k_{17 h}\left(\frac{\bar{w}_{x h}^{\prime}}{\bar{w}_{z h}^{\prime}} \bar{Z}_{w h}-\bar{w}_{x h}\right)\right. \\
& +k_{18 h}\left(\frac{\left.\left.\bar{w}_{x h}^{\prime} \bar{Z}_{w h}-\bar{w}_{z h}\right)\right]}{\bar{w}_{x h}}\right)
\end{aligned}
$$

where $\left(k_{17 h}, k_{18 h}\right)$ are constants. The bias and minimum MSE of $t_{S-13 m}$ at optimum values of constants to first-order approximation are given by:

$$
\begin{array}{r}
\operatorname{Bias}\left(t_{S-13 m}\right) \cong \frac{1}{N} \sum_{h=1}^{L} N_{h} \bar{Y}_{w h}\left[k_{17 h} \bar{X}_{w h} \theta_{h}^{\prime}\right. \\
\left.\left(C_{w_{z h}}^{2}-C_{w_{x} w_{z} h}\right)+k_{18 h} \bar{Z}_{w h} \theta_{h}^{\prime \prime} C_{w_{x h}}^{2}\right]
\end{array}
$$

and:

$$
\begin{aligned}
& M S E\left(t_{S-13 m}\right) \cong \frac{1}{N^{2}} \sum_{h=1}^{L} N_{h}^{2} \bar{Y}_{w h}^{2} \\
& {\left[\theta_{h} C_{w_{y h}}^{2}-\frac{1}{A_{h} B_{h}-E_{h}^{2}}\right.} \\
&\left.\left\{B_{h} C_{h}^{2}+A_{h} D_{h}^{2}-2 D_{h} E_{h} C_{h}\right\}\right],
\end{aligned}
$$

where:

$$
\begin{aligned}
& A_{h}=\theta_{h}^{\prime \prime} C_{w_{x h}}^{2}+\theta_{h}^{\prime} C_{w_{z h}}^{2}, \\
& B_{h}=A_{h}+2 \theta_{h}^{\prime \prime} C_{w_{x} w_{z} h}, \\
& E_{h}=A_{h}+\theta_{h}^{\prime \prime} C_{w_{x} w_{z} h}, \\
& C_{h}=\theta_{h}^{\prime \prime} C_{w_{y h}}+\theta_{h}^{\prime} C_{w_{y} w_{z} h}, \\
& k_{17 h_{o p t}}=\frac{\bar{Y}_{w h}\left(B_{h} C_{h}-D_{h} E_{h}\right)}{\bar{X}_{w h}\left(A_{h} B_{h}-E_{h}^{2}\right)}, \\
& D_{h}=\theta_{h}^{\prime \prime} C_{w_{y} w_{x} h}+\theta_{h}^{\prime} C_{w_{y} w_{z} h}, \\
& k_{18 h_{o p t}}=\frac{\bar{Y}_{w h}\left(A_{h} D_{h}-C_{h} E_{h}\right)}{\bar{Z}_{w h}\left(A_{h} B_{h}-E_{h}^{2}\right)} .
\end{aligned}
$$

14. Based on Khan [24], the exponential-type estimator for population mean in SACS is given by:

$$
\begin{aligned}
& t_{S-14 m}=\frac{1}{N} \sum_{h=1}^{L} N_{h} \\
& {\left[\begin{array}{c}
\bar{w}_{y h} \exp \left(\frac{\bar{w}_{x h}^{\prime}-\bar{w}_{x h}}{\bar{w}_{x h}^{\prime}+\bar{w}_{x h}}\right)^{k_{19 h}} \\
+k_{20 h}\left\{\bar{w}_{x h}^{\prime} \exp \left(\frac{\left.\left.\frac{\bar{Z}_{w h}-\bar{w}_{z h}^{\prime}}{\bar{Z}_{w h}+\bar{w}_{z h}^{\prime}}\right)-\bar{w}_{x h}\right\}}{}\right],\right.
\end{array}\right.}
\end{aligned}
$$

where $\left(k_{19 h}, k_{20 h}\right)$ are constants. The bias and minimum MSE of $t_{S-14 m}$ at optimum values of constants to first-order approximation are given by:

$$
\begin{gathered}
\operatorname{Bias}\left(t_{S-14 m}\right) \cong \frac{1}{N} \sum_{h=1}^{L} N_{h} \bar{Y}_{w h} \\
{\left[\theta _ { h } ^ { \prime \prime } \left\{k_{19 h}\left(\frac{1}{4} C_{w_{x h}}^{2}-\frac{1}{2} C_{w_{y} w_{x} h}\right)\right.\right.} \\
\left.+\frac{1}{8} k_{19 h}^{2} C_{w_{x h}}^{2}\right\}+\theta_{h}^{\prime} k_{20 h} \bar{X}_{w h} \\
\left.\left\{\frac{3}{8} C_{w_{z h}}^{2}+\frac{1}{2} C_{w_{x} w_{z} h}\right\}\right],
\end{gathered}
$$

and:

$$
M S E\left(t_{S-14 m}\right) \cong \frac{1}{N^{2}} \sum_{h=1}^{L} N_{h}^{2} \bar{Y}_{w h}^{2}
$$

$$
\left[\theta_{h} C_{w_{y} h}^{2}-\theta_{h}^{\prime \prime} \rho_{w_{y} w_{x} h}^{2}-\theta_{h}^{\prime} \rho_{w_{y} w_{z} h}^{2}\right]
$$

where:

$$
\begin{aligned}
k_{19 h_{o p t}}= & \frac{4 C_{w_{y h}}}{C_{w_{x h}} C_{w_{z h}}}\left(\frac{1}{2} \rho_{w_{y} w_{x} h} C_{w_{y h}}\right. \\
& \left.+\rho_{w_{y} w_{z} h} C_{w_{x h}}\right), \\
k_{20 h_{o p t}}= & \frac{2 \rho_{w_{y} w_{z} h} C_{w_{y h}}}{\bar{X}_{w h} C_{w_{z h}}} .
\end{aligned}
$$

15. According to Singh et al. [25], the ratio-type estimator for population mean in SACS is given by:

$$
\begin{aligned}
t_{S-15 m_{j}}= & \frac{1}{N} \sum_{h=1}^{L} N_{h} \bar{w}_{y h}\left(\frac{k_{21 h_{x j}} \bar{w}_{x h}^{\prime}+k_{22 h_{x j}} \bar{w}_{x h}}{k_{23 h_{x j}} \bar{w}_{x h}+k_{24 h_{x j}} \bar{w}_{x h}^{\prime}}\right) \\
& \left(\frac{k_{21 h_{z j}} \bar{Z}_{w h}+k_{22 h_{z j}} \bar{w}_{z h}^{\prime}}{k_{23 h_{z j}} \bar{w}_{z h}^{\prime}+k_{24 h_{z j}} \bar{Z}_{w h}}\right),
\end{aligned}
$$

where $\left(k_{21 h_{i j}}, k_{22 h_{i j}}, k_{23 h_{i j}}, k_{24 h_{i j}}, \forall i=x, z\right)$ are constants that assume different values for $j=$ $1,2,3$, as given in Table 2 .

The bias and MSE of $t_{S-15 m_{j}}$ to the first-order approximation are given by: 
Table 2. Combinations of constants for $t_{S-15 m_{j}}$.

\begin{tabular}{ccccccccc}
\hline$j$ & $k_{21 h_{x j}}$ & $k_{22 h_{x j}}$ & $k_{23 h_{x j}}$ & $k_{24 h_{x j}}$ & $k_{21 h_{z j}}$ & $k_{22 h_{z j}}$ & $k_{23 h_{z j}}$ & $k_{24 h_{z j}}$ \\
\hline 1 & $C_{w_{x h}}^{2}$ & $-\rho_{w_{y} w_{x} h}$ & $C_{w_{x h}}^{2}$ & $-\rho_{w_{y} w_{x} h}$ & $C_{w_{z h}}^{2}$ & $-\rho_{w_{y} w_{z} h}$ & $C_{w_{z h}}^{2}$ & $-\rho_{w_{y} w_{z} h}$ \\
2 & $\beta_{2\left(w_{x h}\right)}$ & $-C_{w_{y h}}$ & $\beta_{2\left(w_{x h}\right)}$ & $-C_{w_{y h}}$ & $\beta_{2\left(w_{z h}\right)}$ & $-C_{w_{y h}}$ & $\beta_{2\left(w_{z h}\right)}$ & $-C_{w_{y} h}$ \\
3 & $C_{w_{y h}}$ & $C_{w_{x h}}$ & $C_{w_{y h}}$ & $C_{w_{x h}}$ & $C_{w_{y h}}$ & $C_{w_{z h}}$ & $C_{w_{y} h}$ & $C_{w_{z h}}$ \\
\hline
\end{tabular}

$$
\begin{aligned}
\operatorname{Bias}\left(t_{S-15 m_{j}}\right) \cong \frac{1}{N} \sum_{h=1}^{L} N_{h} \bar{Y}_{w h} \\
\quad-\theta_{h}^{\prime} C_{w_{y} w_{x} h} \delta_{1 h}-\theta_{h}^{\prime} C_{w_{y} w_{z} h} \delta_{3 h} \\
-\theta_{h} C_{w_{y} w_{x} h} q_{x 3 h}+\theta_{h}^{\prime} C_{w_{x} w_{z} h} \\
\left\{q_{x 3 h} q_{z 3 h}+q_{x 4 h} q_{z 3 h}-q_{x 1 h} q_{z 3 h}\right. \\
\left.-q_{x 3 h} q_{z 2 h}-q_{x 4 h} q_{z 2 h}+q_{x 1 h} q_{z 2 h}\right\} \\
+\theta_{h}^{\prime} C_{w_{x h}}^{2}\left\{q_{x 4 h}^{2}-q_{x 1 h} q_{x 4 h}-q_{x 2 h} q_{x 4 h}\right. \\
\left.-q_{x 1 h} q_{x 3 h}+2 q_{x 3 h} q_{x 4 h}\right\}+\theta C_{w_{x h}}^{2} \\
\left\{q_{x 3 h}^{2}-q_{x 2 h} q_{x 3 h}\right\}+\theta_{h}^{\prime} C_{w_{z h}}^{2} \\
\left.\left\{q_{z 3 h}^{2}-q_{z 2 h} q_{z 3 h}\right\}\right]
\end{aligned}
$$

$$
\delta_{3 h}=q_{z 3 h}-q_{z 2 h}
$$

16. Shabbir and Gupta [26] presented the differencecum-exponential ratio-type estimator for population mean in SACS as follows:

$$
\begin{aligned}
t_{S-16 m}= & \frac{1}{N} \sum_{h=1}^{L} N_{h}\left[\left\{k_{25 h} \bar{w}_{y h}+k_{26 h}\right.\right. \\
& \left.\left(\bar{w}_{x h}^{\prime}-\bar{w}_{x h}\right)+k_{27 h}\left(\bar{Z}_{w h}-\bar{w}_{z h}^{\prime}\right)\right\} \\
& \left.\exp \left(\frac{\bar{w}_{x h}^{\prime}-\bar{w}_{x h}}{\bar{w}_{x h}^{\prime}+\bar{w}_{x h}}\right)\right],
\end{aligned}
$$

where $\left(k_{25 h}, k_{26 h}, k_{27 h}\right)$ are constants. The bias and minimum MSE of $t_{S-16 m}$ at optimum values of constants to first-order approximation are given by:

$$
\begin{aligned}
& \operatorname{Bias}\left(t_{S-16 m}\right) \cong \frac{1}{N} \sum_{h=1}^{L} N_{h} \\
& {\left[k_{25 h} \bar{Y}_{w h} \theta_{h}^{\prime \prime}\left\{\frac{3}{8} C_{w_{x h}}^{2}-\frac{1}{2} C_{w_{y} w_{x} h}\right\}\right.} \\
& \left.+k_{26 h} \bar{X}_{w h} \frac{\theta_{h}^{\prime \prime}}{2} C_{w_{x h}}^{2}+\bar{Y}_{w h}\left(k_{25 h}-1\right)\right],
\end{aligned}
$$

and:

$$
\begin{gathered}
M S E\left(t_{S-16 m}\right)_{\min } \cong \frac{1}{N^{2}} \sum_{h=1}^{L} N_{h}^{2} \frac{\bar{Y}_{w h}^{2}}{L_{h}} \\
\left(L_{h}-H_{h}^{2}-\frac{E_{h}^{2} L_{h}}{B_{h}}\right),
\end{gathered}
$$

where:

$$
\left.\left.\left(2 \delta_{1 h} \delta_{3 h}+2 \delta_{2 h} \delta_{3 h h}\right)\right\}\right],
$$

where:

$$
\begin{aligned}
& q_{x 1 h}=\frac{k_{21 h_{x j}}}{k_{21 h_{x j}}+k_{22 h_{x j}}}, \quad q_{x 2 h}=\frac{k_{22 h_{x j}}}{k_{21 h_{x j}}+k_{22 h_{x j}}}, \\
& q_{x 3 h}=\frac{k_{23 h_{x j}}}{k_{23 h_{x j}}+k_{24 h_{x j}}}, \quad q_{x 4 h}=\frac{k_{24 h_{x j}}}{k_{23 h_{x j}}+k_{24 h_{x j}}}, \\
& q_{z 2 h}=\frac{k_{22 h_{z j}}}{k_{21 h_{z j}}+k_{22 h_{z j}}}, \quad q_{z 3 h}=\frac{k_{23 h_{z j}}}{k_{23 h_{z j}}+k_{24 h_{z j}}}, \\
& \delta_{1 h}=q_{x 4 h}-q_{x 1 h}, \quad \delta_{2 h}=q_{x 3 h}-q_{x 2 h},
\end{aligned}
$$

$$
\begin{aligned}
& A_{h}=1+\theta_{h} C_{w_{y} h}^{2}+\theta_{h}^{\prime \prime}\left(C_{w_{x h}}^{2}-2 C_{w_{y} w_{x} h}\right) \\
& F_{h}=\theta_{h}^{\prime \prime}\left(C_{w_{x h}}^{2}-C_{w_{y} w_{x} h}\right) \\
& E_{h}=\frac{\theta_{h}^{\prime \prime}}{2} C_{w_{x h}}^{2}, \quad k_{25 h_{o p t}}=\frac{H_{h}}{L_{h}} \\
& k_{26 h_{o p t}}=\frac{\bar{Y}_{w h}\left(E_{h} L_{h}-F_{h} H_{h}\right)}{\bar{X}_{w h} B_{h} L_{h}} \\
& B_{h}=\theta_{h}^{\prime \prime} C_{w_{x h}}^{2}, \\
& D_{h}=1+\theta_{h}^{\prime \prime}\left(\frac{3}{8} C_{w_{x h}}^{2}-\frac{1}{2} C_{w_{y} w_{x} h}\right)
\end{aligned}
$$




$$
\begin{aligned}
& H_{h}=D_{h}-\frac{E_{h} F_{h}}{B_{h}}, \quad C_{h}=\theta_{h}^{\prime} C_{w_{z h}}^{2}, \\
& G_{h}=\theta_{h}^{\prime} C_{w_{y} w_{z} h}, \quad L_{h}=A_{h}-\frac{F_{h}^{2}}{B_{h}}-\frac{G_{h}^{2}}{C_{h}}, \\
& k_{27 h_{o p t}}=\frac{\bar{Y}_{w h} G_{h} H_{h}}{\bar{Z}_{w h} C_{h} L_{h}} .
\end{aligned}
$$

17. According to Muneer et al. [27], difference-cumexponential estimators for population mean in SACS are given by:

$$
\begin{aligned}
t_{S-17 m}= & \frac{1}{N} \sum_{h=1}^{L} N_{h}\left[\left\{k_{28 h} \bar{w}_{y h}+k_{29 h}\right.\right. \\
& \left.\left(\bar{w}_{x h}^{\prime}-\bar{w}_{x h}\right)\right\}\{2-\exp \\
& \left.\left.\left(\frac{\bar{w}_{z h}^{\prime}-\bar{Z}_{w h}}{\bar{w}_{z h}^{\prime}+\bar{Z}_{w h}}\right)\right\}\right],
\end{aligned}
$$

and:

$$
\begin{aligned}
t_{S-18 m}= & \frac{1}{N} \sum_{h=1}^{L} N_{h}\left[\left\{k_{30 h} \bar{w}_{y h}+k_{31 h}\right.\right. \\
& \left.\left.\left(\bar{w}_{x h}^{\prime}-\bar{w}_{x h}\right)\right\} \exp \left(\frac{\bar{Z}_{w h}-\bar{w}_{z h}^{\prime}}{\bar{Z}_{w h}+\bar{w}_{z h}^{\prime}}\right)\right],
\end{aligned}
$$

where $\left(k_{28 h}, k_{29 h}, k_{30 h}, k_{31 h}\right)$ are constants. The bias and minimum MSE of $t_{S-17 m}$ and $t_{S-18 m}$ at optimum values of constants to first-order of approximation are given by:

$$
\begin{aligned}
& \operatorname{Bias}\left(t_{S-17 m}\right) \cong \frac{1}{N} \sum_{h=1}^{L} N_{h}\left[\bar{Y}_{w h}\left(k_{28 h}-1\right)\right. \\
&\left.+k_{28 h} \bar{Y}_{w h} \theta_{h}^{\prime}\left(\frac{1}{8} C_{w_{z h}}^{2}-\frac{1}{2} C_{w_{y} w_{z} h}\right)\right], \\
& \operatorname{Bias}\left(t_{S-18 m}\right) \cong \frac{1}{N} \sum_{h=1}^{L} N_{h}\left[\bar{Y}_{w h}\left(k_{30 h}-1\right)\right. \\
&\left.+k_{30 h} \bar{Y}_{w h} \theta_{h}^{\prime}\left(\frac{3}{8} C_{w_{z h}}^{2}-\frac{1}{2} C_{w_{y} w_{z} h}\right)\right] \\
& M S E\left(t_{S-17 m}\right)_{\min } \cong \sum_{h=1}^{L} N_{h}^{2}\left[\bar{Y}_{w h}^{2} \frac{M_{1 h}-B_{1 h}^{2}}{M_{1 h}}\right]_{(51)}
\end{aligned}
$$

and:

$$
M S E\left(t_{S-18 m}\right)_{\min } \cong \frac{1}{N^{2}} \sum_{h=1}^{L} N_{h}^{2}\left[\bar{Y}_{w}^{2} \frac{M_{2 h}-B_{2 h}^{2}}{M_{2 h}}\right]_{(52)}
$$

where:

$$
\begin{aligned}
& A_{1 h}=1+\theta_{h} C_{w_{y h}}^{2}+\theta_{h}^{\prime}\left(\frac{1}{2} C_{w_{z h}}^{2}-2 C_{w_{y} w_{z} h}\right), \\
& B_{1 h}=1+\theta_{h}^{\prime}\left(\frac{1}{8} C_{w_{z h}}^{2}-\frac{1}{2} C_{w_{y} w_{z} h}\right) \\
& k_{28 h_{o p t}}=\frac{B_{1 h}}{M_{1 h}}, \\
& A_{2 h}=1+\theta_{h} C_{w_{y h}}^{2}+\theta_{h}^{\prime}\left(C_{w_{z h}}^{2}-2 C_{w_{y} w_{z} h}\right), \\
& B_{2 h}=1+\theta_{h}^{\prime}\left(\frac{3}{8} C_{w_{z h}}^{2}-\frac{1}{2} C_{w_{y} w_{z} h}\right), \\
& D_{h}=C_{w_{x h}}^{2} \\
& F_{h}=C_{w_{y} w_{x} h}, \quad k_{30 h_{o p t}}=\frac{B_{2 h}}{M_{2 h}}, \\
& M_{1 h}=A_{1 h}-\theta_{h}^{\prime \prime} \frac{F_{h}^{2}}{D_{h}}, \\
& k_{29 h_{o p t}}=\frac{\bar{Y}_{w h} F_{h} B_{1 h}}{\bar{X}_{w h} D_{h} M_{1 h}}, \\
& M_{2 h}=A_{2 h}-\theta_{h}^{\prime \prime} \frac{F_{h}^{2}}{D_{h}}, \\
& k_{31 h_{o p t}}=\frac{\bar{Y}_{w h} F_{h} B_{2 h}}{\bar{X}_{w h} D_{h} M_{2 h}} .
\end{aligned}
$$

18. Shabbir [12] found the difference-type estimator for population mean in SACS below:

$$
\begin{aligned}
t_{S-19 m}= & \frac{1}{N} \sum_{h=1}^{L} N_{h}\left[\bar{w}_{y h}+k_{32 h}\left\{\bar{w}_{x h}^{\prime}-\bar{w}_{x h}\right\}\right. \\
& +k_{33 h}\left\{\bar{r}^{\prime}\left(w_{x}\right)_{h}-\bar{r}\left(w_{x}\right)_{h}\right\} \\
& +k_{34 h}\left\{\bar{Z}_{w h}-\bar{w}_{z h}^{\prime}\right\} \\
& \left.+k_{35 h}\left\{\bar{R}\left(w_{z}\right)_{h}-\bar{r}^{\prime}\left(w_{z}\right)_{h}\right\}\right],
\end{aligned}
$$

where $\left(k_{32 h}, k_{33 h}, k_{34 h}, k_{35 h}\right)$ are constants. The bias and minimum MSE of $t_{S-19 m}$ at optimum values of constants to first-order approximation are given by:

$$
\operatorname{Bias}\left(t_{S-19 m}\right)=0,
$$

and:

$$
\begin{gathered}
M S E\left(t_{S-19 m}\right)_{\min } \cong \frac{1}{N^{2}} \sum_{h=1}^{L} N_{h}^{2} \bar{Y}_{w h}^{2} C_{w_{y h}}^{2} \\
{\left[\theta_{h}-\theta_{h}^{\prime \prime} R_{x h}-\theta_{h}^{\prime} R_{z h}\right]}
\end{gathered}
$$

where: 


$$
\begin{aligned}
& R_{x h}= \\
& \frac{\rho_{w_{y} w_{x} h}^{2}+\rho_{w_{y} r\left(w_{x}\right) h}^{2}-2 \rho_{w_{y} w_{x} h} \rho_{w_{y} r\left(w_{x}\right) h} \rho_{w_{x} r\left(w_{x}\right) h}}{1-\rho_{w_{x} r\left(w_{x}\right) h}^{2}} \\
& R_{z h}= \\
& \frac{\rho_{w_{y} w_{z} h}^{2}+\rho_{w_{y} r\left(w_{z}\right) h}^{2}-2 \rho_{w_{y} w_{z} h} \rho_{w_{y} r\left(w_{z}\right) h} \rho_{w_{z} r\left(w_{z}\right) h}}{1-\rho_{w_{z}}^{2} r\left(w_{z}\right) h} \\
& k_{32 h_{o p t}}=\frac{S_{w_{y} h}\left\{\rho_{w_{y} w_{x} h}-\rho_{w_{x} r\left(w_{x}\right) h} \rho_{w_{y} r\left(w_{x}\right) h}\right\}}{S_{w_{y} h}\left(1-\rho_{w_{x} r\left(w_{x} h\right.}^{2}\right)} \\
& k_{33 h_{o p t}}=\frac{S_{w_{y} h}\left\{\rho_{w_{y} r\left(w_{x}\right) h}-\rho_{w_{x} r\left(w_{x}\right) h} \rho_{w_{y} w_{x} h}\right\}}{S_{r\left(w_{x}\right) h}\left(1-\rho_{w_{x} r\left(w_{x}\right) h}^{2}\right)} \\
& k_{34 h_{o p t}}=\frac{S_{w_{y} h}\left\{\rho_{w_{y} w_{z} h}-\rho_{w_{z} r\left(w_{z}\right) h} \rho_{w_{y} r\left(w_{z}\right) h}\right\}}{S_{w_{z} h}\left(1-\rho_{w_{z} r\left(w_{z}\right) h}^{2}\right)} \\
& k_{35 h_{o p t}}=\frac{S_{w_{y} h}\left\{\rho_{w_{y} r\left(w_{z}\right) h}-\rho_{w_{z} r\left(w_{z}\right) h} \rho_{w_{y} w_{z} h}\right\}}{S_{r\left(w_{z}\right) h}\left(1-\rho_{w_{z} r\left(w_{z}\right) h}^{2}\right)} .
\end{aligned}
$$

\subsection{Estimators for population variance}

Some of the existing estimators for population variance using two auxiliary variables are discussed in this section under SACS and SRS. Usual sample variance in SACS is given by:

1. Usual sample variance in SACS is given by:

$$
t_{S-1 v}=\frac{1}{N} \sum_{h=1}^{L} N_{h} s_{w_{y h}}^{2} .
$$

The MSE of $t_{S-1 v}$ to first-order approximation is given by:

$$
M S E\left(t_{S-1 v}\right) \cong \frac{1}{N^{2}} \sum_{h=1}^{L} N_{h}^{2} \theta_{h} S_{w_{y h}}^{4} \lambda_{400 h}^{*} .
$$

2. Usual ratio estimator (using variance of auxiliary variables) for population variance in SACS is given as follows:

$$
t_{S-2 v}=\frac{1}{N} \sum_{h=1}^{L} N_{h} s_{w_{y h}}^{2}\left(\frac{s_{w_{x h}}^{2 \prime}}{s_{w_{x h}}^{2}}\right)\left(\frac{S_{w_{z h}}^{2}}{s_{w_{z h}}^{2 \prime}}\right) .
$$

The bias and MSE of $t_{S-2 v}$ to first-order approximation are given by:

$$
\begin{array}{r}
\operatorname{Bias}\left(t_{S-2 v}\right) \cong \frac{1}{N} \sum_{h=1}^{L} N_{h} S_{w_{y h}}^{2} \\
{\left[\theta_{h}^{\prime \prime}\left(\lambda_{040 h}^{*}-\lambda_{220 h}^{*}\right)+\theta_{h}^{\prime}\left(\lambda_{004 h}^{*}-\lambda_{202 h}^{*}\right)\right],}
\end{array}
$$

and:

$$
\begin{aligned}
& M S E\left(t_{S-2 v}\right) \cong \frac{1}{N^{2}} \sum_{h=1}^{L} N_{h}^{2} S_{w_{y h}}^{4} \\
& {\left[\begin{array}{c}
\theta_{h} \lambda_{400 h}^{*}+\theta_{h}^{\prime \prime}\left(\lambda_{040 h}^{*}-2 \lambda_{220 h}^{*}\right) \\
+\theta_{h}^{\prime}\left(\lambda_{004 h}^{*}-2 \lambda_{202 h}^{*}\right)
\end{array}\right] .}
\end{aligned}
$$

3. Usual ratio estimator (using mean of auxiliary variables) for population variance in SACS is given by:

$$
t_{S-3 v}=\frac{1}{N} \sum_{h=1}^{L} N_{h} s_{w_{y h}}^{2}\left(\frac{\bar{w}_{x h}^{\prime}}{\bar{w}_{x h}}\right)\left(\frac{\bar{Z}_{w h}}{\bar{w}_{z h}^{\prime}}\right)
$$

The bias and MSE of $t_{S-3 v}$ to first-order approximation are given by:

$$
\begin{aligned}
\operatorname{Bias}\left(t_{S-3 v}\right) \cong & \frac{1}{N} \sum_{h=1}^{L} N_{h} S_{w_{y h}}^{2} \\
& {\left[\theta_{h}^{\prime \prime}\left(C_{w_{x h}}^{2}-C_{w_{x h}} \lambda_{210 h}\right)\right.} \\
& \left.+\theta_{h}^{\prime}\left(C_{w_{z h}}^{2}-C_{w_{z h}} \lambda_{201 h}\right)\right],
\end{aligned}
$$

and:

$$
\begin{aligned}
M S E\left(t_{S-3 v}\right) & \cong \frac{1}{N^{2}} \sum_{h=1}^{L} N_{h}^{2} S_{w_{y h}}^{4} \\
& {\left[\begin{array}{c}
\theta_{h} \lambda_{400 h}^{*}+\theta_{h}^{\prime \prime}\left(C_{w_{x h}}^{2}-2 C_{w_{x h}} \lambda_{210 h}\right) \\
+\theta_{h}^{\prime}\left(C_{w_{z h}}^{2}-2 C_{w_{z h}} \lambda_{201 h}\right)
\end{array}\right] . }
\end{aligned}
$$

4. Traditional exponential ratio-type estimator for population variance in SACS is given by:

$$
\begin{aligned}
t_{S-4 v}= & \frac{1}{N} \sum_{h=1}^{L} N_{h} s_{w_{y h}}^{2} \exp \left(\frac{s_{w_{x h}}^{2 \prime}-s_{w_{x h}}^{2}}{s_{w_{x h}}^{2 \prime}+s_{w_{x h}}^{2}}\right) \\
& \exp \left(\frac{S_{w_{z h}}^{2}-s_{w_{z h}}^{2 \prime}}{S_{w_{z h}}^{2}+s_{w_{z h}}^{2 \prime}}\right) .
\end{aligned}
$$

The bias and MSE of $t_{S-4 v}$ to first-order approximation are given by:

$$
\begin{aligned}
\operatorname{Bias}\left(t_{S-4 v}\right) \cong & \frac{1}{N} \sum_{h=1}^{L} N_{h} S_{w_{y h}}^{2} \\
& {\left[\theta_{h}^{\prime \prime}\left(\frac{3 \lambda_{040 h}^{*}}{8}-\frac{\lambda_{220 h}^{*}}{2}\right)\right.} \\
& \left.+\theta_{h}^{\prime}\left(\frac{3 \lambda_{004 h}^{*}}{8}-\frac{\lambda_{202 h}^{*}}{2}\right)\right],
\end{aligned}
$$

and: 


$$
\begin{gathered}
M S E\left(t_{S-4 v}\right) \cong \frac{1}{N^{2}} \sum_{h=1}^{L} N_{h}^{2} S_{w_{y h}}^{4} \\
{\left[\begin{array}{c}
\theta_{h} \lambda_{400 h}^{*}+\theta_{h}^{\prime \prime}\left(\frac{\lambda_{040 h}^{*}}{4}-\lambda_{220 h}^{*}\right) \\
+\theta_{h}^{\prime}\left(\frac{\lambda_{004 h}^{*}}{4}-\lambda_{202 h}^{*}\right)
\end{array}\right] .}
\end{gathered}
$$

5. Traditional difference-type estimator for population variance in SACS is given by:

$$
\begin{aligned}
t_{S-5 v}= & \frac{1}{N} \sum_{h=1}^{L} N_{h}\left[s_{w_{y h}}^{2}+p_{1 h}\left(s_{w_{x h}}^{2 \prime}-s_{w_{x h}}^{2}\right)\right. \\
& \left.+p_{2 h}\left(S_{w_{z h}}^{2}-s_{w_{z h}}^{2 \prime}\right)\right],
\end{aligned}
$$

where $p_{1 h}$ and $p_{2 h}$ are constants. The minimum MSE of $t_{S-5 v}$ at optimum values of constants to first-order approximation is given by:

$$
\begin{aligned}
M S E\left(t_{S-5 v}\right)_{\min } & \cong \frac{1}{N^{2}} \sum_{h=1}^{L} N_{h}^{2} S_{w_{y h}}^{4} \\
& {\left[\theta_{h} \lambda_{400 h}^{*}-\theta_{h}^{\prime \prime}\left(\frac{\lambda_{220 h}^{* 2}}{\lambda_{040 h}^{*}}\right)-\theta_{h}^{\prime}\left(\frac{\lambda_{202 h}^{* 2}}{\lambda_{004 h}^{*}}\right)\right], }
\end{aligned}
$$

where:

$$
p_{1 h_{o p t}}=\frac{S_{w_{y h}}^{2} \lambda_{220 h}^{*}}{S_{w_{x h}}^{2} \lambda_{040 h}^{*}}, \quad p_{2 h_{o p t}}=\frac{S_{w_{y h}}^{2} \lambda_{202 h}^{*}}{S_{w_{z h}}^{2} \lambda_{004 h}^{*}} .
$$

6. Singh et al. [28] presented the exponential ratiotype estimator for population variance in SACS below:

$$
\begin{aligned}
t_{S-6 v}= & \frac{1}{N} \sum_{h=1}^{L} N_{h} s_{w_{y h}}^{2}\left[p_{3 h} \exp \left(\frac{s_{w_{x h}}^{2^{\prime}}-s_{w_{x h}}^{2}}{s_{w_{x h}}^{2^{\prime}}+s_{w_{x h}}^{2}}\right)\right. \\
& \left.+\left(1-p_{3 h}\right) \exp \left(\frac{S_{w_{z h}}^{2}-s_{w_{z h}}^{2^{\prime}}}{S_{w_{z h}}^{2}+s_{w_{z h}}^{2^{\prime}}}\right)\right]
\end{aligned}
$$

where $p_{3 h}$ is a constant. The bias and minimum MSE of $t_{S-6 v}$ at optimum values of constants to first-order approximation are given by:

$$
\begin{gathered}
\operatorname{Bias}\left(t_{S-6 v}\right) \cong \frac{1}{N} \sum_{h=1}^{L} N_{h} S_{w_{y h}}^{2} \\
{\left[p _ { 3 h } \left\{\theta_{h}^{\prime \prime}\left(\frac{3}{8} \lambda_{04000 h}^{*}-\frac{1}{2} \lambda_{22000 h}^{*}\right)\right.\right.} \\
\left.-\theta_{h}^{\prime}\left(\frac{3}{8} \lambda_{00040 h}^{*}-\frac{1}{2} \lambda_{20020 h}^{*}\right)\right\} \\
\left.+\theta_{h}^{\prime}\left(\frac{3}{8} \lambda_{00040 h}^{*}-\frac{1}{2} \lambda_{20020 h}^{*}\right)\right]
\end{gathered}
$$

and:

$$
M S E\left(t_{S-6 v}\right)_{\min } \cong \frac{1}{N^{2}} \sum_{h=1}^{L} N_{h}^{2} S_{w_{y h}}^{4}\left(D_{h}-\frac{B_{h}^{2}}{A_{h}}\right)_{(71)}
$$

where:

$$
\begin{aligned}
& A_{h}=\frac{\theta_{h}^{\prime \prime}}{4} \lambda_{04000 h}^{*}+\frac{\theta_{h}^{\prime}}{4} \lambda_{00040 h}^{*}, \quad p_{3 h_{\text {opt }}}=\frac{-B_{h}}{A_{h}} \\
& B_{h}=\theta_{h}^{\prime}\left(\frac{1}{2} \lambda_{20020 h}^{*}-\frac{1}{4} \lambda_{00040 h}^{*}\right)-\frac{\theta_{h}^{\prime \prime}}{2} \lambda_{22000 h}^{*}, \\
& D_{h}=\theta_{h} \lambda_{40000 h}^{*}+\theta_{h}^{\prime}\left(\frac{1}{4} \lambda_{00040 h}^{*}-\lambda_{20020 h}^{*}\right) .
\end{aligned}
$$

7. As proposed by Singh and Solanki [29], the ratiotype estimator for population variance in SACS is given by:

$$
\begin{aligned}
t_{S-7 v_{j}}= & \frac{1}{N} \sum_{h=1}^{L} N_{h} s_{w_{y h}}^{2}\left(\frac{p_{4 h_{x j}} s_{w_{x h}}^{2^{\prime}}+p_{5 h_{x j}}}{p_{4 h_{x j}} s_{w_{x}}^{2}+p_{5 h_{x j}}}\right) \\
& \left(\frac{p_{4 h_{z j}} S_{w_{z h}}^{2}+p_{5 h_{z j}}}{p_{4 h_{z j}} s_{w_{z}}^{2^{\prime}}+p_{5 h_{z j}}}\right),
\end{aligned}
$$

where $\left(p_{4 h_{x j}}, p_{5 h_{x j}}, p_{4 h_{z j}}, p_{5 h_{z j}}\right)$ are constants that assume different values for $j=1,2,3,4$ as given in Table 3.

The bias and MSE of $t_{S-7 v_{j}}$ to first-order approximation are given by:

$$
\begin{gathered}
\operatorname{Bias}\left(t_{S-7 v_{j}}\right) \cong \frac{1}{N} \sum_{h=1}^{L} N_{h} S_{w_{y h}}^{2} \\
{\left[\theta_{h}^{\prime \prime}\left\{Q_{x h}^{2} \lambda_{04000 h}^{*}-Q_{x h} \lambda_{22000 h}^{*}\right\}\right.} \\
\left.+\theta_{h}^{\prime}\left\{Q_{z h}^{2} \lambda_{00040 h}^{*}-Q_{z h} \lambda_{20020 h}^{*}\right\}\right],
\end{gathered}
$$

and:

$$
\begin{array}{r}
M S E\left(t_{S-7 v_{j}}\right) \cong \frac{1}{N^{2}} \sum_{h=1}^{L} N_{h}^{2} S_{w_{y h}}^{4} \\
{\left[\theta_{h}^{\prime \prime}\left\{Q_{x h}^{2} \lambda_{04000 h}^{*}-2 Q_{x h} \lambda_{22000 h}^{*}\right\}\right.}
\end{array}
$$

Table 3. Combinations of constants for $t_{S-7 v_{j}}$.

\begin{tabular}{ccccc}
\hline$j$ & 1 & 2 & 3 & 4 \\
\hline$p_{4 h_{x j}}$ & 1 & 1 & $C_{w_{x h}}$ & $\beta_{2\left(w_{x h}\right)}$ \\
$p_{5 h_{x j}}$ & $C_{w_{x h}}$ & $\beta_{2\left(w_{x h}\right)}$ & $\beta_{2\left(w_{x h}\right)}$ & $C_{w_{x h}}$ \\
$p_{4 h_{z j}}$ & 1 & 1 & $C_{w_{x h}}$ & $\beta_{2\left(w_{z h}\right)}$ \\
$p_{5 h_{z j}}$ & $C_{w_{z h}}$ & $\beta_{2\left(w_{z h}\right)}$ & $\beta_{2\left(w_{x h}\right)}$ & $C_{w_{z h}}$ \\
\hline
\end{tabular}




$$
\begin{aligned}
& +\theta_{h} \lambda_{40000 h}^{*}+\theta_{h}^{\prime}\left\{Q_{z h}^{2} \lambda_{00040 h}^{*}\right. \\
& \left.\left.-2 Q_{z h} \lambda_{20020 h}^{*}\right\}\right]
\end{aligned}
$$

where:

$$
\begin{aligned}
Q_{x h} & =\frac{p_{4 h_{x j}} S_{w_{x h}}^{2}}{p_{4 h_{x j}} S_{w_{x h}}^{2}+p_{5 h_{x j}}}, \\
Q_{z h} & =\frac{p_{4 h_{z j}} S_{w_{z h}}^{2}}{p_{4 h_{z j}} S_{w_{z h}}^{2}+p_{5 h_{z j}}} .
\end{aligned}
$$

8. Olufadi and Kadilar [30] presented the ratio-type estimator for population variance in SACS as follows:

$$
t_{S-8 v}=\frac{1}{N} \sum_{h=1}^{L} N_{h} s_{w_{y h}}^{2}\left(\frac{s_{w_{x h}}^{2^{\prime}}}{s_{w_{x h}}^{2}}\right)^{p_{6 h}}\left(\frac{S_{w_{z h}}^{2}}{s_{w_{z h}}^{2^{\prime}}}\right)^{p_{7 h}}
$$

where $p_{6 h}$ and $p_{7 h}$ are constants. The bias and minimum MSE of $t_{S-8 v}$ at optimum values of constants to first-order approximation are given by:

$$
\begin{aligned}
\operatorname{Bias}\left(t_{S-8 v}\right) \cong \frac{1}{N} \sum_{h=1}^{L} N_{h} S_{w_{y h}}^{2} \\
{\left[\theta_{h}^{\prime \prime}\left\{\frac{p_{6 h}\left(p_{6 h}+1\right)}{2} \lambda_{04000 h}^{*}-p_{6 h} \lambda_{22000 h}^{*}\right\}\right.} \\
\left.+\theta_{h}^{\prime}\left\{\frac{p_{7 h}\left(p_{7 h}+1\right)}{2} \lambda_{00040 h}^{*}-p_{7 h} \lambda_{20020 h}^{*}\right\}\right],
\end{aligned}
$$

and:

$$
\begin{aligned}
M S E\left(t_{S-8 v}\right)_{\min } & \cong \frac{1}{N^{2}} \sum_{h=1}^{L} N_{h}^{2} S_{w_{y h}}^{4} \\
& {\left[\theta_{h} \lambda_{40000 h}^{*}-\theta_{h}^{\prime \prime} \frac{\lambda_{22000 h}^{* 2}}{\lambda_{04000 h}^{*}}-\theta_{h}^{\prime} \frac{\lambda_{20020 h}^{* 2}}{\lambda_{00040 h}^{*}}\right] }
\end{aligned}
$$

where:

$$
p_{6 h_{o p t}}=\frac{\lambda_{22000 h}^{*}}{\lambda_{04000 h}^{*}}, \quad p_{7 h_{o p t}}=\frac{\lambda_{20020 h}^{*}}{\lambda_{00040 h}^{*}} .
$$

9. Amin et al. [31] presented the ratio-type estimators for population variance in SACS below:

$$
\begin{aligned}
t_{S-9 v}= & \frac{1}{N} \sum_{h=1}^{L} N_{h} s_{w_{y h}}^{2} \\
& \left\{\frac{s_{w_{x h}}^{2^{\prime}}}{p_{8 h} s_{w_{x h}}^{2^{\prime}}+\left(1-p_{8 h}\right) s_{w_{x h}}^{2}}\right\}^{p_{9 h}} \\
& \left\{\frac{S_{w_{z h}}^{2}}{p_{10 h} S_{w_{z h}}^{2}+\left(1-p_{10 h}\right) s_{w_{z h}}^{2^{\prime}}}\right\}^{p_{11 h}},
\end{aligned}
$$

and:

$$
t_{S-10 v}=\frac{1}{N} \sum_{h=1}^{L} N_{h} p_{12 h} s_{w_{y h}}^{2}\left(\frac{s_{w_{x h}}^{2^{\prime}}}{s_{w_{x h}}^{2}}\right)\left(\frac{S_{w_{z h}}^{2}}{s_{w_{z h}}^{2^{\prime}}}\right) .
$$

The minimum MSE values for $t_{S-9 v}$ and $t_{S-10 v}$ at optimum values of constants to first-order approximation are given by:

$$
\begin{aligned}
M S E\left(t_{S-9 v}\right)_{\min } \cong & \frac{1}{N^{2}} \sum_{h=1}^{L} N_{h}^{2} S_{w_{y h}}^{4} \\
& {\left[\theta_{h} \lambda_{40000 h}^{*}-\theta_{h}^{\prime \prime} \frac{\lambda_{22000 h}^{* 2}}{\lambda_{04000 h}^{*}}-\theta_{h}^{\prime} \frac{\lambda_{20020 h}^{* 2}}{\lambda_{00040 h}^{*}}\right], }
\end{aligned}
$$

and:

$$
M S E\left(t_{S-10 v}\right)_{\min } \cong \frac{1}{N^{2}} \sum_{h=1}^{L} N_{h}^{2} S_{w_{y h}}^{4}\left(1-\frac{M_{1 h}^{2}}{L_{1 h}}\right)
$$

where:

$$
\begin{aligned}
& p_{9 h}\left(1-p_{8 h}\right)_{o p t}=\frac{\lambda_{22000 h}^{*}}{\lambda_{04000 h}^{*}}, \\
& p_{11 h}\left(1-p_{10 h}\right)_{o p t}=\frac{\lambda_{20020 h}^{*}}{\lambda_{00040 h}^{*}}, \\
& L_{1 h}=1+\theta_{h} \lambda_{40000 h}^{*}+\theta_{h}^{\prime \prime}\left(3 \lambda_{04000 h}^{*}-4 \lambda_{22000 h}^{*}\right) \\
& +\theta_{h}^{\prime}\left(3 \lambda_{00040 h}^{*}-4 \lambda_{20020 h}^{*}\right) \\
& M_{1 h}=1+\theta_{h}^{\prime \prime}\left(\lambda_{04000 h}^{*}-\lambda_{22000 h}^{*}\right) \\
& \quad+\theta_{h}^{\prime}\left(\lambda_{00040 h}^{*}-\lambda_{20020 h}^{*}\right) \\
& =\frac{M_{1 h}}{L_{1 h}} .
\end{aligned}
$$

\section{Proposed estimators}

\subsection{Difference-type estimator}

The following difference-type estimator is proposed for general parameters under SACS:

$$
\begin{aligned}
\hat{\tau}_{S-(\alpha, \beta) y P 1} & =\frac{1}{N} \sum_{h=1}^{L} N_{h}\left[\hat{\tau}_{(\alpha, \beta) y h}+f_{1 h}\left\{\bar{w}_{x h}^{\prime}-\bar{w}_{x h}\right\}\right. \\
& +f_{2 h}\left\{s_{w_{x h}^{\prime}}^{2^{\prime}}-s_{w_{x h}}^{2}\right\}+\left\{\bar{r}^{\prime}\left(w_{x}\right)_{h}-\bar{r}\left(w_{x}\right)_{h}\right\} \\
& +f_{3 h}\left\{\bar{Z}_{w h}-\bar{w}_{z h}^{\prime}\right\}+f_{4 h}\left\{S_{w_{z h}}^{2}-s_{w_{z h}}^{2^{\prime}}\right\} \\
& \left.+\left\{\bar{R}\left(w_{z}\right)_{h}-\bar{r}^{\prime}\left(w_{z}\right)_{h}\right\}\right],
\end{aligned}
$$

where $f_{i h}(i=1,2,3,4)$ are constants whose values are to be determined. Estimators for population mean $\left(t_{S-P 1 m}\right)$ and variance $\left(t_{S-P 1 v}\right)$ can be obtained by substituting $(\alpha=1, \beta=0)$ and $(\alpha=0, \beta=2)$ in Eq. (82), respectively. Rewriting Eq. (82) in terms of errors, we get: 


$$
\begin{aligned}
\hat{\tau}_{S-(\alpha, \beta) y P 1}- & \tau_{(\alpha, \beta) y} \cong \frac{1}{N} \sum_{h=1}^{L} N_{h} \tau_{(\alpha, \beta) y h} \\
& {\left[\left\{\alpha \zeta_{0 h}+\frac{\beta}{2} \zeta_{3 h}+\frac{\alpha(\alpha-1)}{2} \zeta_{0 h}^{2}\right.\right.} \\
& \left.+\frac{\beta(\beta-2)}{8} \zeta_{3 h}^{2}+\frac{\alpha \beta}{2} \zeta_{0 h} \zeta_{3 h}\right\} \\
& +f_{1 h} \bar{X}_{w h}\left(\zeta_{1 h}^{\prime}-\zeta_{1 h}\right) \\
& +f_{2 h} S_{w_{x h}}^{2}\left(\zeta_{4 h}^{\prime}-\zeta_{4 h}\right)+\bar{R}\left(w_{x}\right)_{h}\left(\zeta_{6 h}^{\prime}-\zeta_{6 h}\right) \\
& -f_{3 h} \bar{Z}_{w h}^{\prime} \zeta_{2 h}-f_{4 h} S_{w_{z h}}^{2} \zeta_{5 h}^{\prime} \\
& \left.-\bar{R}\left(w_{z}\right)_{h} \zeta_{7 h}^{\prime}\right]
\end{aligned}
$$

Taking expectations of both sides, we get:

$$
\begin{aligned}
\operatorname{Bias}\left(\hat{\tau}_{S-(\alpha, \beta) y P 1}\right) \cong \frac{1}{N} \sum_{h=1}^{L} N_{h} \tau_{(\alpha, \beta) y} \theta_{h} \\
\left\{\frac{\alpha(\alpha-1)}{2} C_{w_{y h}}^{2}+\frac{\beta(\beta-2)}{8} \lambda_{40000 h}^{*}\right. \\
\left.+\frac{\alpha \beta}{2} C_{w_{y h}} \lambda_{30000 h}\right\} .
\end{aligned}
$$

Bias of the proposed estimator for population mean $\left(t_{S-P 1 m}\right)$ and variance $\left(t_{S-P 1 v}\right)$ can be obtained by substituting $(\alpha=1, \beta=0)$ and $(\alpha=0, \beta=2)$ in Eq. (84), respectively. Squaring Eq. (83) and considering first-order approximation, we get:

$$
\begin{aligned}
{\left[\hat{\tau}_{S-(\alpha, \beta) y P 1}-\right.} & \left.\tau_{(\alpha, \beta) y}\right]^{2} \cong \frac{1}{N^{2}} \sum_{h=1}^{L} N_{h}^{2} \\
& {\left[\tau_{(\alpha, \beta) y h}\left(\alpha \zeta_{0 h}+\frac{\beta}{2} \zeta_{3 h}\right)\right.} \\
& +f_{1 h} \bar{X}_{w h}\left(\zeta_{1 h}^{\prime}-\zeta_{1 h}\right)+f_{2 h} S_{w_{x h}}^{2} \\
& \left(\zeta_{4 h}^{\prime}-\zeta_{4 h}\right)+\bar{R}\left(w_{x}\right)_{h}\left(\zeta_{6 h}^{\prime}-\zeta_{6 h}\right) \\
& -f_{3 h} \bar{Z}_{w h}^{\prime} \zeta_{2 h}-f_{4 h} S_{w_{z h}}^{2} \zeta_{5 h}^{\prime} \\
& \left.-\bar{R}\left(w_{z}\right)_{h} \zeta_{7 h}^{\prime}\right]^{2} .
\end{aligned}
$$

Taking expectations of both sides, we get:

$$
\begin{aligned}
M S E & \left(\hat{\tau}_{S-(\alpha, \beta) y P 1}\right) \cong \frac{1}{N^{2}} \sum_{h=1}^{L} N_{h}^{2} \tau_{(\alpha, \beta) y h}^{2} \theta_{h} A_{y h} \\
& +\theta_{h}^{\prime}\left\{f_{3 h}^{2} A_{z h}+f_{4 h}^{2} B_{z h}-2 f_{3 h} D_{z h}-2 f_{4 h} E_{z h}\right.
\end{aligned}
$$

$$
\begin{aligned}
& \left.+2 f_{3 h} f_{4 h} F_{z h}+G_{z h}\right\}+\theta_{h}^{\prime \prime}\left\{f_{1 h}^{2} A_{x h}+f_{2 h}^{2} B_{x h}\right. \\
& \left.-2 f_{1 h} D_{x h}-2 f_{2 h} E_{x h}+2 f_{1 h} f_{2 h} F_{x h}+G_{x h}\right\},
\end{aligned}
$$

where:

$$
\begin{aligned}
& A_{y h}=\alpha^{2} C_{w_{y h}}^{2}+\frac{\beta^{2}}{4} \lambda_{40000 h}^{*}+\alpha \beta C_{w_{y h}} \lambda_{30000 h}, \\
& A_{x h}=\bar{X}_{w h}^{2} C_{w_{x h}}^{2}, \\
& B_{x h}=S_{w_{x h}}^{4} \lambda_{04000 h}^{*}, \\
& F_{x h}=\bar{X}_{w h} S_{w_{x h}}^{2} C_{w_{x h}} \lambda_{03000 h}, \\
& D_{x h}=\tau_{(\alpha, \beta) y h} \bar{X}_{w h}\left(\alpha C_{w_{y} w_{x} h}+\frac{\beta}{2} C_{w_{x h}} \lambda_{21000 h}\right) \\
& -\bar{X}_{w h} \bar{R}\left(w_{x}\right)_{h} C_{w_{x} r\left(w_{x}\right) h}, \\
& E_{x h}=\tau_{(\alpha, \beta) y h} S_{w_{x h}}^{2}\left(\alpha C_{w_{y h}} \lambda_{12000 h}+\frac{\beta}{2} \lambda_{22000 h}^{*}\right) \\
& -S_{w_{x h}}^{2} \bar{R}\left(w_{x}\right)_{h} C_{r\left(w_{x h}\right)} \lambda_{02100 h}, \\
& G_{x h}=\bar{R}\left(w_{x}\right)_{h}^{2} C_{r\left(w_{x h}\right)}^{2}-2 \tau_{(\alpha, \beta) y h} \bar{R}\left(w_{x}\right)_{h} \\
& \left(\alpha C_{w_{y} r\left(w_{x}\right) h}+\frac{\beta}{2} C_{r\left(w_{x h}\right)} \lambda_{20100 h}\right), \\
& A_{z h}=\bar{Z}_{w h}^{2} C_{w_{z h}}^{2}, \\
& B_{z h}=S_{w_{z h}}^{4} \lambda_{00040 h}^{*}, \\
& F_{z h}=\bar{Z}_{w h} S_{w_{z h}}^{2} C_{w_{z h}} \lambda_{00030 h}, \\
& D_{z h}=\tau_{(\alpha, \beta) y h} \bar{Z}_{w h}\left(\alpha C_{w_{y} w_{z} h}+\frac{\beta}{2} C_{w_{z h}} \lambda_{20010 h}\right) \\
& -\bar{Z}_{w h} \bar{R}\left(w_{z}\right)_{h} C_{w_{z} r\left(w_{z}\right) h}, \\
& E_{z h}=\tau_{(\alpha, \beta) y h} S_{w_{z h}}^{2}\left(\alpha C_{w_{y h}} \lambda_{10020 h}+\frac{\beta}{2} \lambda_{20020 h}^{*}\right) \\
& -S_{w_{z h}}^{2} \bar{R}\left(w_{z}\right)_{h} C_{r\left(w_{z h}\right)} \lambda_{00021 h}, \\
& G_{z h}=\bar{R}\left(w_{z}\right)_{h}^{2} C_{r\left(w_{z h}\right)}^{2}-2 \tau_{(\alpha, \beta) y h} \bar{R}\left(w_{z}\right)_{h} \\
& \left(\alpha C_{w_{y} r\left(w_{z}\right) h}+\frac{\beta}{2} C_{r\left(w_{z h}\right)} \lambda_{20001 h}\right) .
\end{aligned}
$$

From (Eq. 86), the optimum values of $f_{i h}(i=1,2,3,4)$ are:

$$
\begin{aligned}
f_{1 h_{o p t}} & =\frac{B_{x h} D_{x h}-E_{x h} F_{x h}}{A_{x h} B_{x h}-F_{x h}^{2}}, \\
f_{2 h_{o p t}} & =\frac{A_{x h} E_{x h}-F_{x h} D_{x h}}{A_{x h} B_{x h}-F_{x h}^{2}},
\end{aligned}
$$




$$
\begin{aligned}
f_{3 h_{o p t}} & =\frac{B_{z h} D_{z h}-E_{z h} F_{z h}}{A_{z h} B_{z h}-F_{z h}^{2}}, \\
f_{4 h_{o p t}} & =\frac{A_{z h} E_{z h}-F_{z h} D_{z h}}{A_{z h} B_{z h}-F_{z h}^{2}} .
\end{aligned}
$$

By substituting optimum values of $f_{i h}(i=1,2,3,4)$ in Eq. (86), the minimum MSE of the proposed differencetype estimator for general parameters is obtained as follows:

$$
\begin{aligned}
\operatorname{MSE}\left(\hat{\tau}_{S-(\alpha, \beta) y P 1}\right)_{\min } \cong \frac{1}{N^{2}} \sum_{h=1}^{L} N_{h}^{2} \\
{\left[\tau_{(\alpha, \beta) y h}^{2} \theta_{h} A_{y h}+\theta_{h}^{\prime \prime} Q_{x h}+\theta_{h}^{\prime} Q_{z h}\right], }
\end{aligned}
$$

where:

$$
\begin{aligned}
& Q_{i h}=G_{i h}+\frac{2 D_{i h} E_{i h} F_{i h}-B_{i h} D_{i h}^{2}-A_{i h} E_{i h}^{2}}{A_{i h} B_{i h}-F_{i h}^{2}}, \\
& \forall i=x, z .
\end{aligned}
$$

Minimum MSE of the proposed estimator for population mean $\left(t_{S-P 1 m}\right)$ and variance $\left(t_{S-P 1 v}\right)$ can be obtained by substituting $(\alpha=1, \beta=0)$ and $(\alpha=$ $0, \beta=2$ ) in Eq. (87), respectively.

\subsection{Difference-cum-exponential-ratio-type estimator}

The following difference-cum-exponential-ratio-type estimator is proposed for general parameters under SACS:

$$
\begin{aligned}
\hat{\tau}_{S-(\alpha, \beta) y P 2} & =\frac{1}{N} \sum_{h=1}^{L} N_{h}\left[\left\{g_{1 h} \hat{\tau}_{(\alpha, \beta) y h}\right.\right. \\
& \left.+g_{2 h}\left(\bar{w}_{x h}^{\prime}-\bar{w}_{x h}\right)+g_{3 h}\left(\bar{Z}_{w h}-\bar{w}_{z h}^{\prime}\right)\right\} \\
& \exp \left(\frac{s_{w_{x h}^{\prime}}^{2^{\prime}}-s_{w_{x h}}^{2}}{s_{w_{x h}}^{2^{\prime}}+s_{w_{x h}}^{2}}\right) \\
& \left.\exp \left(\frac{\bar{r}^{\prime}\left(w_{x}\right)_{h}-\bar{r}\left(w_{x}\right)_{h}}{\bar{r}^{\prime}\left(w_{x}\right)_{h}+\bar{r}\left(w_{x}\right)_{h}}\right)\right]
\end{aligned}
$$

where $g_{i h}(i=1,2,3)$ are constants whose values are to be determined. Estimators for population mean $\left(t_{S-P 2 m}\right)$ and population variance $\left(t_{S-P 2 v}\right)$ can be obtained by substituting ( $\alpha=1, \beta=0)$ and $(\alpha=0, \beta=$ 2 ) in Eq. (88), respectively. Rewriting Eq. (88) in terms of errors and considering first order approximation, we get:

$$
\begin{aligned}
\hat{\tau}_{S-(\alpha, \beta) y P 2} \cong \frac{1}{N} \sum_{h=1}^{L} N_{h}\left[\left\{g_{1 h} \tau_{(\alpha, \beta) y h}\right.\right. \\
\left(1+\alpha \zeta_{0 h}+\frac{\beta}{2} \zeta_{3 h}+\frac{\alpha(\alpha-1)}{2} \zeta_{0 h}^{2}\right.
\end{aligned}
$$

$$
\begin{aligned}
& \left.+\frac{\alpha \beta}{2} \zeta_{0 h} \zeta_{3 h}+\frac{\beta(\beta-2)}{8} \zeta_{3 h}^{2}\right) \\
& \left.+g_{2 h} \bar{X}_{w h}\left[\zeta_{1 h}^{\prime}-\zeta_{1 h}\right]-g_{3 h} \bar{Z}_{w h}^{\prime} \zeta_{2 h}\right\} \\
& \left\{1+\frac{\zeta_{4 h}^{\prime}-\zeta_{4 h}}{2}+\frac{3\left(\zeta_{4 h}^{2}-\zeta_{4 h}^{2^{\prime}}\right)}{8}\right\} \\
& \left\{1+\frac{\zeta_{6 h}^{\prime}-\zeta_{6 h}}{2}+\frac{3\left(\zeta_{6 h}^{2}-\zeta_{6 h}^{2^{\prime}}\right)}{8}\right\}
\end{aligned}
$$

Taking expectations of both sides, we get:

$$
\begin{aligned}
& \operatorname{Bias}\left(\hat{\tau}_{S-(\alpha, \beta) y P 2}\right) \cong \frac{1}{N} \sum_{h=1}^{L} N_{h} \\
& \quad\left[g_{1 h} \tau_{(\alpha, \beta) y h}\left(\theta_{h}^{\prime \prime} Q_{1 h}+\theta_{h} T_{1 h}\right)+g_{2 h} \bar{X}_{w h} \frac{\theta_{h}^{\prime \prime}}{2} U_{1 h}\right. \\
& \left.\quad+\tau_{(\alpha, \beta) y h}\left(g_{1 h}-1\right)\right]
\end{aligned}
$$

where:

$$
\begin{aligned}
Q_{1 h}= & \frac{3}{8} \lambda_{04000 h}^{*}+\frac{3}{8} C_{r\left(w_{x h}\right)}^{2}-\frac{\alpha}{2} \\
& \left(C_{w_{y h}} \lambda_{12000 h}+C_{w_{y} r\left(w_{x}\right) h}\right) \\
& +\frac{1}{4} C_{r\left(w_{x h}\right)} \lambda_{02100 h}-\frac{\beta}{4}\left(\lambda_{22000 h}^{*}\right. \\
& \left.+C_{r\left(w_{x h}\right)} \lambda_{20100 h}\right), \\
T_{1 h}= & \frac{\alpha(\alpha-1)}{2} C_{w_{y h}}^{2}+\frac{\beta(\beta-2)}{8} \lambda_{40000 h}^{*} \\
+ & \frac{\alpha \beta}{2} C_{w_{y h}} \lambda_{30000 h}, \\
U_{1 h}= & C_{w_{x h}} \lambda_{03000 h}+C_{w_{x} r\left(w_{x}\right) h} .
\end{aligned}
$$

Bias of the estimator for population mean $\left(t_{S-P 2 m}\right)$ and population variance $\left(t_{S-P 2 v}\right)$ can be obtained by substituting $(\alpha=1, \beta=0)$ and $(\alpha=0, \beta=2)$ in Eq. (90), respectively. Squaring Eq. (89) and considering first-order approximation, we have:

$$
\begin{aligned}
& {\left[\hat{\tau}_{S-(\alpha, \beta) y P 2}-\tau_{(\alpha, \beta) y}\right]^{2} \cong \frac{1}{N^{2}} \sum_{h=1}^{L} N_{h}^{2}} \\
& {\left[g _ { 1 h } ^ { 2 } \tau _ { ( \alpha , \beta ) y h } ^ { 2 } \left\{\frac{\zeta_{4 h}^{\prime}-\zeta_{4 h}}{2}+\frac{\zeta_{6 h}^{\prime}-\zeta_{6 h}}{2}\right.\right.} \\
& \left.\quad+\alpha \zeta_{0 h}+\frac{\beta}{2} \zeta_{3 h}\right\}^{2}-2 g_{h} g_{3 h} \bar{X}_{w h} \bar{Z}_{w h}
\end{aligned}
$$




$$
\begin{aligned}
& \left\{\zeta_{1 h}^{\prime} \zeta_{2 h}^{\prime}-\zeta_{1 h}^{\prime} \zeta_{2 h}\right\}+g_{2 h}^{2} \bar{X}_{w h}^{2}\left\{\zeta_{1 h}^{\prime}-\zeta_{1 h}\right\}^{2} \\
& +g_{3 h}^{2} \bar{Z}_{w h}^{2} \zeta_{4 h}^{2^{\prime}}+\tau_{(\alpha, \beta) y h}^{2}\left(g_{1 h}-1\right)^{2} \\
& -2 g_{1 h} g_{3 h} \tau_{(\alpha, \beta) y h} \bar{Z}_{w h}\left\{\alpha \zeta_{0 h}^{\prime} \zeta_{2 h}\right. \\
& \left.+\frac{\beta}{2} \zeta_{3 h} \zeta_{2 h}^{\prime}\right\}+2 g_{1 h} g_{2 h} \bar{X}_{w h} \tau_{(\alpha, \beta) y h} \\
& \left\{\frac{\zeta_{1 h} \zeta_{4 h}-\zeta_{4 h} \zeta_{1 h}^{\prime}}{2}+\frac{\zeta_{1 h} \zeta_{6 h}-\zeta_{6 h} \zeta_{1 h}^{\prime}}{2}\right. \\
& +\alpha\left(\zeta_{0 h} \zeta_{1 h}^{\prime}-\zeta_{0 h} \zeta_{1 h}\right)+\frac{\beta}{2} \\
& \left.\left.+\frac{\zeta_{1 h} \zeta_{6 h}-\zeta_{1 h} \zeta_{6 h}^{\prime}}{2}\right\}\right] \\
& \left.\left.+\zeta_{3 h} \zeta_{1 h}^{\prime}-\zeta_{3 h} \zeta_{1 h}\right)\right\}+2 g_{1 h}\left(g_{1 h}-1\right) \\
& +\frac{\alpha(\alpha-1)}{2} \zeta_{0 h}^{2}+\frac{\beta(\beta-2)}{8} \zeta_{3 h}^{2}+\frac{\alpha \beta}{2} \zeta_{0 h} \zeta_{3 h} \\
& +\frac{\beta}{4}\left(\zeta_{3 h} \zeta_{4 h}^{\prime}-\zeta_{3 h} \zeta_{4 h}+\zeta_{3 h} \zeta_{6 h}^{\prime}-\zeta_{3 h} \zeta_{6 h}\right) \\
& \left(\zeta_{0 h} \zeta_{4 h}^{\prime}-\zeta_{0 h}^{2^{\prime}} \zeta_{4 h}+\zeta_{0 h} \zeta_{6 h}^{\prime}-\zeta_{0 h} \zeta_{6 h}\right) \\
& \left.8 \zeta_{4 h}^{2^{\prime}}\right) \\
& +\frac{\alpha}{2}
\end{aligned}
$$

Taking expectations of both sides, we have:

$$
\begin{aligned}
M S E & \left(\hat{\tau}_{S-(\alpha, \beta) y P 2}\right) \cong \frac{1}{N^{2}} \sum_{h=1}^{L} N_{h}^{2} \\
& {\left[\tau_{(\alpha, \beta) y h}^{2}+g_{1 h}^{2} A_{1 h}+g_{2 h}^{2} B_{1 h}+g_{3 h}^{2} D_{1 h}\right.} \\
& -2 g_{1 h} E_{1 h}-2 g_{2 h} F_{1 h}+2 g_{1 h} g_{2 h} H_{1 h} \\
& \left.-2 g_{1 h} g_{3 h} J_{1 h}\right]
\end{aligned}
$$

where:

$$
A_{1 h}=\tau_{(\alpha, \beta) y h}^{2}\left(1+\theta_{h} a_{x h}+\theta_{h}^{\prime \prime} a_{z h}\right),
$$

$$
\begin{aligned}
& E_{1 h}=\tau_{(\alpha, \beta) y h}^{2}\left(1+\theta_{h} e_{x h}+\theta_{h}^{\prime \prime} e_{z h}\right), \\
& a_{x h}=\left(2 \alpha^{2}-\alpha\right) C_{w_{y h}}^{2}+\frac{\left(\beta^{2}-\beta\right)}{2} \lambda_{04000 h}^{*} \\
& +2 \alpha \beta C_{w_{y h}} \lambda_{30000 h}, \\
& B_{1 h}=\theta_{h}^{\prime \prime} b_{x h}, \\
& a_{z h}=C_{r\left(w_{x h}\right)} \lambda_{02100 h}-2 \alpha\left(C_{w_{y h}} \lambda_{12000 h}+C_{w_{y h} r\left(w_{x h}\right)}\right) \\
& \lambda_{04000 h}^{*}-\beta\left(\lambda_{22000 h}^{*}+C_{r\left(w_{x h}\right)} \lambda_{20100 h}\right)+C_{r\left(w_{x h}\right)}^{2}, \\
& J_{1 h}=\theta_{h}^{\prime} j_{z h} \\
& e_{x h}=\frac{\alpha(\alpha-1)}{2} C_{w_{y h}}^{2}+\frac{\beta(\beta-2)}{8} \lambda_{40000 h}^{*} \\
& +\frac{\alpha \beta}{2} C_{w_{y h}} \lambda_{30000 h} \\
& b_{x h}=\bar{X}_{w h}^{2} C_{w_{x h}}^{2}, \\
& j_{z h}=\tau_{(\alpha, \beta) y h} \bar{Z}_{w h}\left(\alpha C_{w_{y} w_{z} h}+\frac{\beta}{2} C_{w_{z h}} \lambda_{20010 h}\right), \\
& D_{1 h}=\theta_{h}^{\prime} d_{z h}, \quad d_{z h}=\bar{Z}_{w h}^{2} C_{w_{z h}}^{2}, \\
& e_{z h}=\frac{3}{8} \lambda_{04000 h}^{*}+\frac{1}{4} C_{r\left(w_{x h}\right)} \lambda_{02100 h} \\
& -\frac{\alpha}{2}\left(C_{w_{y h}} \lambda_{12000 h}+C_{w_{y} r\left(w_{x}\right) h}\right) \\
& +\frac{3}{8} C_{r\left(w_{x h}\right)}^{2}-\frac{\beta}{4}\left(\lambda_{22000 h}^{*}+C_{r\left(w_{x h}\right)} \lambda_{20100 h}\right), \\
& h_{x h}=\tau_{(\alpha, \beta) y h} \bar{X}_{w h}\left(C_{w_{x h}} \lambda_{03000 h}+C_{w_{x} r\left(w_{x}\right) h}\right. \\
& \left.-\alpha C_{w_{y} w_{x} h}-\frac{\beta}{2} C_{w_{x h}} \lambda_{21000 h}\right)
\end{aligned}
$$

$$
\begin{aligned}
& f_{x h}=\frac{\tau_{(\alpha, \beta) y h} \bar{X}_{w h}}{2}\left(C_{w_{x h}} \lambda_{03000 h}+C_{w_{x} r\left(w_{x}\right) h}\right), \\
& F_{1 h}=\theta_{h}^{\prime \prime} f_{x h}, \quad H_{1 h}=\theta_{h}^{\prime \prime} h_{x h} .
\end{aligned}
$$

From Eq. (92), the optimum values of $g_{i h},(i=1,2,3)$ are as follows:

$$
\begin{aligned}
g_{1 h} & =\frac{L_{1 h}}{M_{1 h}}, \quad g_{2 h}=\frac{F_{1 h} M_{1 h}-H_{1 h} L_{1 h}}{B_{1 h} M_{1 h}}, \\
g_{3 h} & =\frac{J_{1 h} L_{1 h}}{D_{1 h} M_{1 h}}
\end{aligned}
$$

where: 


$$
\begin{aligned}
& M_{1 h}=A_{1 h}-\frac{H_{1 h}^{2}}{B_{1 h}}-\frac{J_{1 h}^{2}}{D_{1 h}}, \\
& L_{1 h}=E_{h}-\frac{F_{1 h} H_{1 h}}{B_{1 h}} .
\end{aligned}
$$

By substituting optimum values of $g_{i h}(i=1,2,3)$ in Eq. (92), the minimum MSE of the proposed exponential ratio-type estimator for general parameters is as follows:

$$
\begin{gathered}
M S E\left(\hat{\tau}_{S-(\alpha, \beta) y P 2}\right)_{\min } \cong \frac{1}{N^{2}} \sum_{h=1}^{L} N_{h}^{2} \\
{\left[\tau_{(\alpha, \beta) y h}^{2}-\frac{1}{M_{1 h}}\left\{L_{1 h}^{2}+\frac{F_{1 h}^{2} M_{1 h}}{B_{1 h}}\right\}\right] .}
\end{gathered}
$$

The minimum MSE of the proposed exponential ratiotype estimator for population mean $\left(t_{S-P 2 m}\right)$ and variance $\left(t_{S-P 2 v}\right)$ can be obtained by substituting ( $\alpha=$ $1, \beta=0)$ and $(\alpha=0, \beta=2)$ in Eq. (93), respectively.

\section{Numerical study}

Data of teals from Smith et al. [32] are considered to make a numerical comparison between the existing and the proposed estimators. The data of Bluewinged teal is used as a study variable for stratum 1 and data of Green-winged teal is used as a study variable for stratum 2 . Auxiliary variables $\left(x_{h}\right.$ and $\left.z_{h}\right)$ are generated using the concept given by Dryver and Chao [33] and Chao et al. [34] as follows:

$$
x_{i}= \begin{cases}y_{i} * \operatorname{Poi}(600)+\epsilon_{i} & \text { if } y_{i}<100 \\ y_{i} & \text { otherwise }\end{cases}
$$

where $\epsilon_{i} \sim N\left(0, y_{i}\right)$ and $P o i$ represents random generation from Poisson distribution. Data statistics at different levels of correlation are given below:

1. $N=400, N_{1}=200, N_{2}=200, n_{1}=20, n_{2}=20$, $n_{1}^{\prime}=50, n_{2}^{\prime}=50, E\left(v_{1}\right)=38, \rho_{w_{y} w_{x} 1}=0.42$, $\rho_{w_{y} w_{x} 2}=0.47, \rho_{w_{y} w_{z} 1}=0.40, \rho_{w_{y} w_{z} 2}=0.41$, $\rho_{w_{x} w_{z} 1}=0.998, \rho_{w_{x} w_{z} 2}=0.987, \bar{Y}_{w 1}=70.60485$, $\bar{Y}_{w 2}=12.01, S_{w_{y 1}}^{2}=130872.4, S_{w_{y 2}}^{2}=12816.53$, $E\left(v_{2}\right)=22, \bar{X}_{w 1}=367.81, \bar{X}_{w 2}=47.64, S_{w_{x} 1}^{2}=$ $1473602, S_{w_{x} 2}^{2}=116807.1, \bar{Z}_{w 1}=391.035, \bar{Z}_{w 2}=$ $59.24, S_{w_{z} 1}^{2}=1713164, S_{w_{z} 2}^{2}=172156$.

2. $\rho_{w_{y} w_{x} 1}=0.66, \rho_{w_{y} w_{x} 2}=0.61, \rho_{w_{y} w_{z} 1}=0.59$, $\rho_{w_{y} w_{z} 2}=0.58, \rho_{w_{x} w_{z} 1}=0.993, \rho_{w_{x} w_{z} 2}=0.989$, $\bar{Y}_{w 1}=70.60485, \bar{Y}_{w 2}=12.01, S_{w_{y 1}}^{2}=130872.4$, $S_{w_{y 2}}^{2}=12816.53, \bar{X}_{w 1}=208.79, \bar{X}_{w 2}=35.33$, $S_{w_{x} 1}^{2}=439839.1, S_{w_{x} 2}^{2}=55797.76, \bar{Z}_{w 1}=232.095$, $\bar{Z}_{w 2}=36.255, S_{w_{z} 1}^{2}=563897.9, S_{w_{z} 2}^{2}=60042$.

3. $\rho_{w_{y} w_{x} 1}=0.88, \rho_{w_{y} w_{x} 2}=0.83, \rho_{w_{y} w_{z} 1}=0.84$, $\rho_{w_{y} w_{z} 2}=0.78, \rho_{w_{x} w_{z} 1}=0.995, \rho_{w_{x} w_{z} 2}=0.996$, $\bar{Y}_{w 1}=70.60485, \bar{Y}_{w 2}=12.01, S_{w_{y 1}}^{2}=130872.4$,

$$
\begin{aligned}
& S_{w_{y 2}}^{2}=12816.53, \bar{X}_{w 1}=125.165, \bar{X}_{w 2}=22.22 \\
& S_{w_{x} 1}^{2}=191574.7, S_{w_{x} 2}^{2}=22789.27, \bar{Z}_{w 1}=138.9 \\
& \bar{Z}_{w 2}=24.545, S_{w_{z} 1}^{2}=216293.5, S_{w_{z}}^{2}=27145 .
\end{aligned}
$$

4. $\rho_{w_{y} w_{x} 1}=0.92, \rho_{w_{y} w_{x} 2}=0.94, \rho_{w_{y} w_{z} 1}=0.89$, $\rho_{w_{y} w_{z} 2}=0.83, \quad \rho_{w_{x} w_{z} 1}=0.997, \quad \rho_{w_{x} w_{z} 2}=$ $0.964, \bar{Y}_{w 1}=70.60485, \bar{Y}_{w 2}=12.01, S_{w_{y 1}}^{2}=$ $130872.4, S_{w_{y 2}}^{2}=12816.53, \bar{X}_{w 1}=115.055, \bar{X}_{w 2}=$ $18.485, S_{w_{x} 1}^{2}=174073.3, S_{w_{x} 2}^{2}=17558.42, \bar{Z}_{w 1}=$ 121.36, $\bar{Z}_{w 2}=20.845, S_{w_{z} 1}^{2}=186673.5, S_{w_{z} 2}^{2}=$ 21254 .

For the data sets discussed above, Absolute Relative Bias (ARB) and Percent Relative Efficiency (PRE) are calculated for the existing and proposed estimators. Results of ARB and PRE of the existing and proposed estimators for population mean are presented in Tables 4 and 5. Similarly, results of ARB and Relative Efficiency (RE) for the existing and proposed estimators for population variance are given in Tables 6 and 7 . Results presented in Tables $4-7$ reveal that for the proposed difference cum exponential-ratio-type estimator for population mean and variance, ARB decreases upon an increase in the correlation between the study and auxiliary variables. The proposed differencetype estimator population mean $\left(t_{S-P 1 m}\right)$ and for population variance $\left(t_{S-P 1 v}\right)$ is unbiased. Thus, ARB remains zero at all correlation levels.

The proposed difference-cum-exponential-ratiotype estimator for population mean $\left(t_{S-P 2 m}\right)$ is more efficient when the correlation between the study and auxiliary variables is low or moderate. The proposed difference type estimator for population mean $\left(t_{S-P 1 m}\right)$ outperforms all other estimators when the correlation is high. When the correlation between the study and auxiliary variables is low or moderate, $t_{S-16 m}$ is most efficient among all existing estimators. Thus, the comparison between $t_{S-P 2 m}$ and $t_{S-16 m}$ is given in Figure 1(a). At high correlation levels, $t_{S-19 m}$ outperforms all existing estimators. Thus, the comparison between $t_{S-P 1 m}$ and $t_{S-19 m}$ is given in Figure 1(b). Finally, the comparison between $t_{S-P 1 m}$ and $t_{S-P 2 m}$ is given in Figure $1(\mathrm{c})$.

The proposed difference-cum-exponential-ratiotype estimator for population variance $t_{S-P 2 v}$ is more efficient when the correlation between the study and auxiliary variables is low or moderate. The proposed difference-type estimator for population variance $t_{S-P 1 v}$ outperforms all other estimators when the correlation is high. When the correlation between the study and the auxiliary variables is low or moderate, $t_{S-10 v}$ is most efficient among all existing estimators. Thus, the comparison between $t_{S-P 2 v}$ and $t_{S-10 v}$ is given in Figure 2(a). At high levels of correlation, $t_{S-9 v}$ performs better than all existing estimators. Thus, the comparison between $t_{S-P 1 v}$ and $t_{S-9 v}$ is given in 
Table 4. Absolute Relative Bias (ARB) of different estimators for population mean.

\begin{tabular}{|c|c|c|c|c|}
\hline \multirow{3}{*}{$\begin{array}{c}\text { ARB } \\
\text { Estimators }\end{array}$} & \multicolumn{4}{|c|}{$\begin{array}{c}\left(\rho_{w y w x h 1}, \rho_{w y w z h 1}:\right. \\
\left.\rho_{w y w x h 2}, \rho_{w y w z h 2}\right)\end{array}$} \\
\hline & $(0.42,0.40:$ & $(0.66,0.59:$ & $(0.88,0.84:$ & $(0.92,0.89:$ \\
\hline & $0.47,0.41)$ & $0.61,0.58)$ & $0.83,0.78)$ & $0.94,0.83)$ \\
\hline$t_{S-1 m}$ & 0.0000 & 0.0000 & 0.0000 & 0.0000 \\
\hline$t_{S-2 m}$ & 0.5515 & 0.1493 & 0.2145 & 0.3068 \\
\hline$t_{S-3 m}$ & 0.1023 & 0.0807 & 0.2696 & 0.3321 \\
\hline$t_{S-4 m}$ & 0.0000 & 0.0000 & 0.0000 & 0.0000 \\
\hline$t_{S-5 m}$ & 1.3852 & 1.1827 & 0.6127 & 1.0998 \\
\hline$t_{S-6 m}$ & 0.1654 & 0.0622 & 0.1285 & 0.1892 \\
\hline$t_{S-7 m}$ & 0.1654 & 0.0622 & 0.1285 & 0.1892 \\
\hline$t_{S-8 m}$ & 0.6076 & 0.2005 & 0.3638 & 0.5285 \\
\hline$t_{S-9 m}$ & 27.8491 & 16.4717 & 82.6546 & 88.8825 \\
\hline$t_{S-10 m_{j=1}}$ & 0.3695 & 0.0134 & 0.3697 & 0.4766 \\
\hline$t_{S-10 m_{j=2}}$ & 0.0173 & 0.1951 & 0.3524 & 0.3556 \\
\hline$t_{S-10 m_{j=3}}$ & 0.5488 & 0.1454 & 0.2200 & 0.3122 \\
\hline$t_{S-10 m_{j=4}}$ & 0.2729 & 0.0480 & 0.3832 & 0.4903 \\
\hline$t_{S-10 m_{j=5}}$ & 0.5371 & 0.1295 & 0.2454 & 0.3470 \\
\hline$t_{S-10 m_{j=6}}$ & 0.5482 & 0.1451 & 0.2203 & 0.3127 \\
\hline$t_{S-10 m_{j=7}}$ & 0.3110 & 0.0370 & 0.3789 & 0.4862 \\
\hline$t_{S-10 m_{j=8}}$ & 0.5514 & 0.1492 & 0.2151 & 0.3072 \\
\hline$t_{S-10 m_{j=9}}$ & 0.5515 & 0.1493 & 0.2148 & 0.3068 \\
\hline$t_{S-10 m_{j=10}}$ & 0.0000 & 0.0000 & 0.0000 & 0.0000 \\
\hline$t_{S-11 m}$ & 0.1004 & 0.1621 & 0.5210 & 0.6334 \\
\hline$t_{S-12 m}$ & 0.6897 & 0.7432 & 0.9310 & 1.0603 \\
\hline$t_{S-13 m}$ & 3.7853 & 1.0862 & 10.7265 & 14.3532 \\
\hline$t_{S-14 m}$ & 3534.36 & 5727.64 & 10347.7 & 14189.8 \\
\hline$t_{S-15 m_{j=1}}$ & 0.5934 & 0.2024 & 0.1532 & 0.2412 \\
\hline$t_{S-15 m_{j=2}}$ & 2.8973 & 7.2896 & 3.1225 & 1.4372 \\
\hline$t_{S-15 m_{j=3}}$ & 0.3445 & 0.4902 & 0.7428 & 0.8782 \\
\hline$t_{S-16 m}$ & 0.4559 & 0.4245 & 0.2804 & 0.1944 \\
\hline$t_{S-17 m}$ & 0.6559 & 0.6233 & 0.5304 & 0.4702 \\
\hline$t_{S-18 m}$ & 0.6000 & 0.5664 & 0.4558 & 0.3608 \\
\hline$t_{S-19 m}$ & 0.0000 & 0.0000 & 0.0000 & 0.0000 \\
\hline $\mathbf{t}_{\mathrm{S}-\mathrm{P} 1 \mathrm{~m}}$ & 0.0000 & 0.0000 & 0.0000 & 0.0000 \\
\hline $\mathbf{t}_{\mathrm{S}-\mathbf{P} 2 \mathrm{~m}}$ & 0.2978 & 0.3798 & 0.2527 & 0.1442 \\
\hline
\end{tabular}

Figure 2(b). Thus, the comparison between $t_{S-P 1 v}$ and $t_{S-P 2 v}$ is given in Figure 2(c).

\section{Conclusion}

In this article, difference-type and difference-cumexponential-ratio-type estimators were recommended for general parameters under stratified adaptive cluster sampling. Estimators were proposed using two auxiliary variables. The proposed estimators utilized auxiliary information in terms of mean, variance, and ranks of auxiliary variates in the $h$ th stratum. Based on the numerical study, it became clear that the proposed estimators for population mean were more efficient than the usual mean, ratio, exponentialratio, difference estimator, and estimators of Gupta and Shabbir [14], Singh et al. [15], Choudhury and Singh [16], Hamad et al. [17], Chutiman [18], Yadav et al. [19], Vishwakarma and Gangele [21], Singh and Khalid [22], Khan and Al-Hossain [23], Khan [24], 
Table 5. Percent Relative Efficiency (PRE) of different estimators for population mean.

\begin{tabular}{|c|c|c|c|c|}
\hline \multirow{3}{*}{$\begin{array}{c}\text { PRE } \\
\text { Estimators }\end{array}$} & \multicolumn{4}{|c|}{$\begin{array}{c}\left(\rho_{w y w x h 1}, \rho_{w y w z h 1}:\right. \\
\left.\rho_{w y w x h 2}, \rho_{w y w z h 2}\right)\end{array}$} \\
\hline & $(0.42,0.40:$ & $(0.66,0.59:$ & $(0.88,0.84:$ & $(0.92,0.89:$ \\
\hline & $0.47,0.41)$ & $0.61,0.58)$ & $0.83,0.78)$ & $0.94,0.83)$ \\
\hline$t_{S-1 m}$ & 165.4901 & 165.4901 & 165.4901 & 165.4901 \\
\hline$t_{S-2 m}$ & 187.4728 & 276.0105 & 584.5970 & 786.8074 \\
\hline$t_{S-3 m}$ & 198.6005 & 236.4534 & 314.0641 & 344.9164 \\
\hline$t_{S-4 m}$ & 200.5705 & 277.1917 & 674.2123 & 983.4634 \\
\hline$t_{S-5 m}$ & 216.6821 & 406.8355 & 1261.3110 & 1707.0440 \\
\hline$t_{S-6 m}$ & 200.5705 & 277.1917 & 674.2123 & 983.4634 \\
\hline$t_{S-7 m}$ & 200.5705 & 277.1917 & 674.2123 & 983.4634 \\
\hline$t_{S-8 m}$ & 300.6597 & 834.2741 & $*$ & $*$ \\
\hline$t_{S-9 m}$ & 107.9679 & 108.2773 & 89.7860 & 81.7906 \\
\hline$t_{S-10 m_{j=1}}$ & 188.8910 & 276.3462 & 561.1049 & 726.3032 \\
\hline$t_{S-10 m_{j=2}}$ & 190.9492 & 271.1515 & 477.9477 & 550.4525 \\
\hline$t_{S-10 m_{j=3}}$ & 187.5180 & 276.0182 & 583.6020 & 784.7066 \\
\hline$t_{S-10 m_{j=4}}$ & 189.5292 & 276.3007 & 554.8144 & 706.2154 \\
\hline$t_{S-10 m_{j=5}}$ & 187.6124 & 276.0827 & 580.1901 & 775.3147 \\
\hline$t_{S-10 m_{j=6}}$ & 187.5244 & 276.0207 & 583.5318 & 784.4774 \\
\hline$t_{S-10 m_{j}=7}$ & 189.3103 & 276.3136 & 556.6877 & 713.5036 \\
\hline$t_{S-10 m_{j=8}}$ & 187.4735 & 276.0113 & 584.5723 & 786.7348 \\
\hline$t_{S-10 m_{j}=9}$ & 187.4728 & 276.0105 & 584.5970 & 786.8074 \\
\hline$t_{S-10 m_{j=10}}$ & 165.4901 & 165.4901 & 165.4901 & 165.4901 \\
\hline$t_{S-11 m}$ & 199.0499 & 245.0164 & 376.5730 & 433.6570 \\
\hline$t_{S-12 m}$ & 190.5514 & 217.4764 & 264.4360 & 279.9786 \\
\hline$t_{S-13 m}$ & 176.5711 & 195.3811 & 201.5196 & 200.5391 \\
\hline$t_{S-14 m}$ & 200.5705 & 277.1917 & 674.2123 & 983.4634 \\
\hline$t_{S-15 m_{j=1}}$ & 182.4002 & 274.0209 & 651.5288 & 922.5695 \\
\hline$t_{S-15 m_{j}=2}$ & 102.7571 & 81.9119 & 256.3006 & 458.5207 \\
\hline$t_{S-15 m_{j=3}}$ & 182.8062 & 197.0932 & 209.2839 & 208.3950 \\
\hline$t_{S-16 m}$ & 460.1971 & 545.3373 & 1036.8820 & 1402.9160 \\
\hline$t_{S-17 m}$ & 402.2027 & 455.2807 & 642.7281 & 735.3240 \\
\hline$t_{S-18 m}$ & 420.8380 & 477.4118 & 683.2319 & 790.3534 \\
\hline$t_{S-19 m}$ & 252.2743 & 284.3129 & 1046.1300 & 1812.2380 \\
\hline $\mathbf{t}_{\mathrm{S}-\mathrm{P} 1 \mathrm{~m}}$ & 303.3198 & 348.0587 & 5152.0570 & 17687.0400 \\
\hline $\mathbf{t}_{\mathrm{S}-\mathrm{P} 2 \mathrm{~m}}$ & 488.7737 & 547.2316 & 1219.2100 & 1823.2250 \\
\hline
\end{tabular}

Shabbir and Gupta [13], Singh et al. [25], Shabbir and Gupta [26], Muneer et al. [27], Shabbir [12], and Qureshi et al. [20] under Stratified Adaptive Cluster Sampling (SACS).

Likewise, the proposed estimators for population variance were found more efficient than usual variance, ratio, exponential-ratio, difference estimator, and es- timators of Singh et al. [28], Singh and Solanki [29], Olufadi and Kadilar [30], and Noor-Ul-Amin et al. [31] under SACS.

At a low or moderate correlation, the proposed difference-cum-exponential-ratio-type estimator was the most efficient one; in addition, at a high correlation between the study and auxiliary vari- 
Table 6. Absolute Relative Bias (ARB) of different estimators for population variance.

\begin{tabular}{ccccc}
\hline ARB & \multicolumn{5}{c}{$\left(\rho_{w y w x h 1}, \rho_{w y w z h 1}:\right.$} \\
\hline Estimators & $(0.42,0.40:$ & $(0.66,0.59:$ & $(0.88,0.84:$ & $(0.92,0.89:$ \\
& $0.47,0.41)$ & $0.61,0.58)$ & $0.83,0.78)$ & $0.94,0.83)$ \\
\hline$t_{S-1 v}$ & 0.0000 & 0.0000 & 0.0000 & 0.0000 \\
$t_{S-2 v}$ & 1.9450 & 0.6879 & 0.2080 & 0.2778 \\
$t_{S-3 v}$ & 0.6095 & 0.1882 & 0.2036 & 0.3146 \\
$t_{S-4 v}$ & 0.6790 & 0.1487 & 0.2942 & 0.3701 \\
$t_{S-5 v}$ & 0.0000 & 0.0000 & 0.0000 & 0.0000 \\
$t_{S-6 v}$ & 0.3290 & 0.0590 & 0.2392 & 0.3182 \\
$t_{S-7 v_{j=1}}$ & 1.9448 & 0.6878 & 0.2084 & 0.2784 \\
$t_{S-7 v_{j=2}}$ & 1.9422 & 0.6858 & 0.2106 & 0.2826 \\
$t_{S-7 v_{j=3}}$ & 1.9446 & 0.6876 & 0.2084 & 0.2785 \\
$t_{S-7 v_{j=4}}$ & 1.9450 & 0.6880 & 0.2081 & 0.2778 \\
$t_{S-8 v}$ & 0.1664 & 0.1730 & 0.1234 & 0.1610 \\
$\mathbf{t}_{\mathbf{S}-\mathbf{P 1 v}}$ & 0.0000 & 0.0000 & 0.0000 & 0.0000 \\
$\mathbf{t}_{\mathbf{S}-\mathbf{P 2 v}}$ & 0.3023 & 0.3897 & 0.2751 & 0.1722 \\
\hline
\end{tabular}

Table 7. Relative Efficiency (RE) of different estimators for population variance.

\begin{tabular}{ccccc}
\hline RE & \multicolumn{5}{c}{$\left(\rho_{w y w x h 1}, \rho_{w y w z h 1}:\right.$} \\
& \multicolumn{5}{c}{$\left.\rho_{w y w x h 2}, \rho_{w y w z h 2}\right)$} \\
\hline Estimators & $(0.42,0.40:$ & $(0.66,0.59:$ & $(0.88,0.84:$ & $(0.92,0.89:$ \\
& $0.47,0.41)$ & $0.61,0.58)$ & $0.83,0.78)$ & $0.94,0.83)$ \\
\hline$t_{S-1 v}$ & 21.37727 & 21.37727 & 21.37727 & 21.37727 \\
$t_{S-2 v}$ & 15.71694 & 30.27605 & 176.42810 & 347.89820 \\
$t_{S-3 v}$ & 23.25597 & 34.30783 & 72.82164 & 96.14209 \\
$t_{S-4 v}$ & 20.77428 & 28.58016 & 51.40028 & 59.05843 \\
$t_{S-5 v}$ & 21.92377 & 31.33513 & 263.27930 & 603.49040 \\
$t_{S-6 v}$ & 21.88700 & 26.52829 & 36.26399 & 38.08119 \\
$t_{S-7 v_{j=1}}$ & 15.71700 & 30.27619 & 176.41870 & 347.85300 \\
$t_{S-7 v_{j=2}}$ & 15.71754 & 30.27718 & 176.37890 & 347.60100 \\
$t_{S-7 v_{j=3}}$ & 15.71704 & 30.27624 & 176.41580 & 347.83580 \\
$t_{S-7 v_{j=4}}$ & 15.71694 & 30.27605 & 176.42760 & 347.89630 \\
$t_{S-8 v}$ & 21.92377 & 31.33513 & 263.27930 & 603.49040 \\
$t_{S-9 v}$ & 21.92377 & 31.33513 & 263.27930 & 603.49040 \\
$t_{S-10 v}$ & 33.84675 & 61.00437 & 189.48520 & 402.68180 \\
$\mathbf{t}_{\mathbf{S}-\mathbf{P 1 v}}$ & 45.67044 & 37.93838 & $\mathbf{7 6 4 . 3 9 1 9 0}$ & $\mathbf{2 0 7 7 . 8 1 7 0 0}$ \\
$\mathbf{t}_{\mathbf{S}-\mathbf{P 2 v}}$ & $\mathbf{5 8 . 3 6 2 2 9}$ & $\mathbf{6 5 . 4 4 7 2 4}$ & 141.49770 & 203.16210 \\
\hline
\end{tabular}

ables, the proposed difference-type estimator was of highest Percent Relative Efficiency (PRE) among all other estimators. Thus, the proposed difference-type and difference-cum-exponential-ratio-type estimators are suggested to make an efficient estimation of population mean and variance under rare and clustered pop- ulations like pollution concentration, drug addiction, and epidemiological studies of AIDS and HIV.

The present study considered ranks of the auxiliary variables for efficient estimation of general parameters under SACS design. Zamanzade and Vock [35] found that when actual quantification of the concomi- 


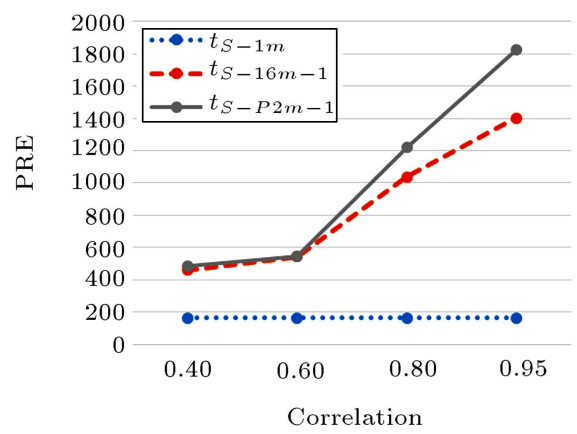

(a) $t_{S-P 2 m}$

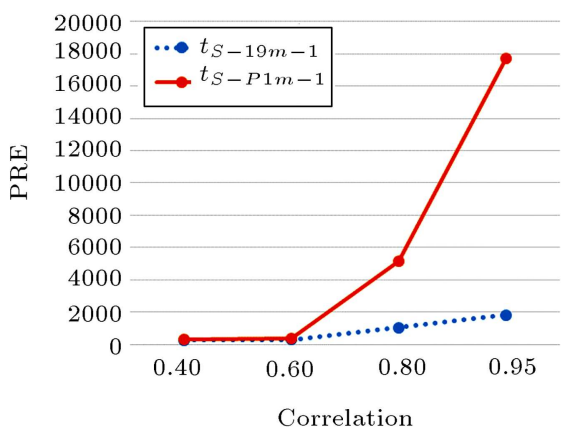

(b) $t_{S-P 1 m}$

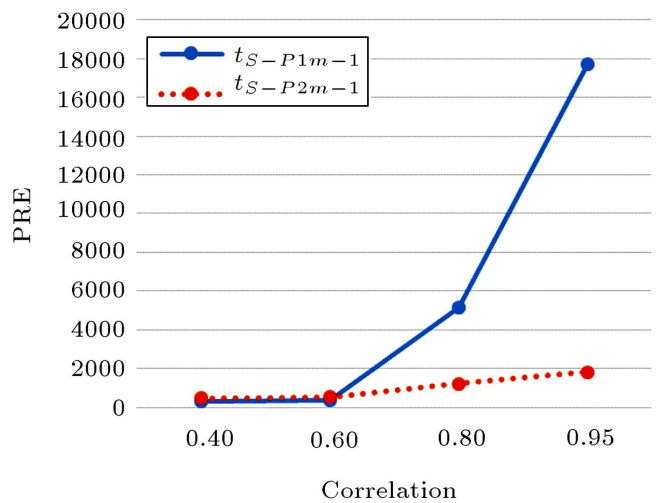

(c) Comparison of the proposed estimators for mean

Figure 1. Percent Relative Efficiency (PRE) of estimators for mean in Stratified Adaptive Cluster Sampling (SACS).

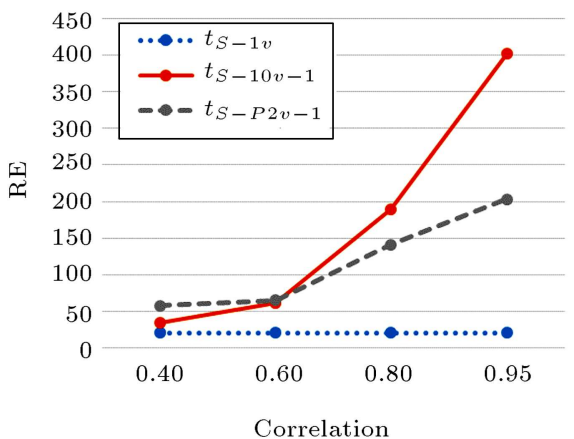

(a) $t_{S-P 2} v$

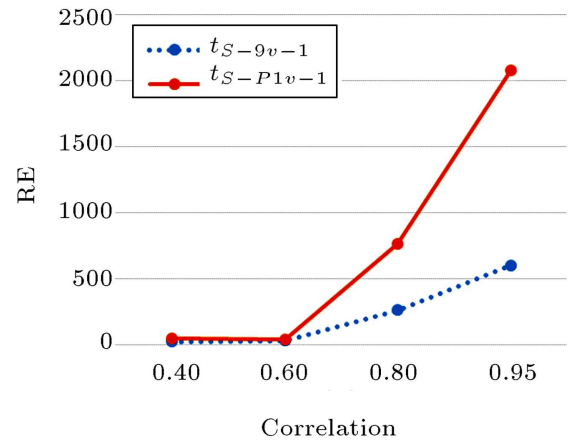

(b) $t_{S-P 1} v$

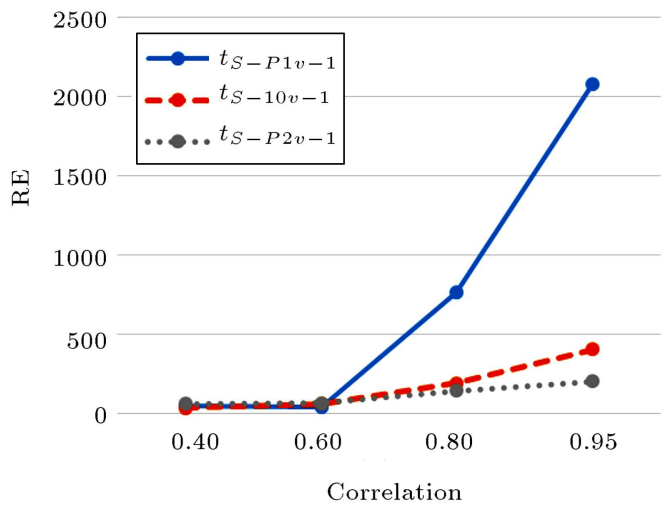

(c) Comparison of the proposed estimators for variance

Figure 2. Relative Efficiency (RE) of estimators for variance in Stratified Adaptive Cluster Sampling (SACS). 
tant variable was available, the ranked set sampling would be more efficient than usual double sampling. A rewarding area for further study is to incorporate ranked set sampling under Adaptive Cluster Sampling (ACS) and SACS designs.

\section{Acknowledgement}

The authors are thankful to the Editor-in-Chief, Prof. S.T.A. Niaki, and anonymous referees for their valuable suggestions that helped to improve the article.

\section{References}

1. Thompson, S. K. "Adaptive cluster sampling", J. Am. Stat. Assoc., 85(412), pp. 1050-1059 (1990).

2. Chutiman, N., Chiangpradit, M., and Suraphee, S. "Ratio estimator in adaptive cluster sampling based on ranked set", Adv. Appl. Stat., 49(2), pp. 105-116 (2016).

3. Gattone, S.A., Giordani, P., Battista, T.D., and Fortuna, F. "Adaptive cluster double sampling with post stratification with application to an epiphytic lichen community", Environ. Ecol. Stat., 25(1), pp. 125-138 (2018).

4. Yasmeen, U. and Thompson, M. "Variance estimation in adaptive cluster sampling", Communications in Statistics-Theory and Methods, https://doi. org/10.1080/03610926.2019.1576890 (2019).

5. Qureshi, M.N., Khalil, S., and Hanif, M. "Joint influence of exponential ratio and exponential product estimator for the estimation clustered population mean in adaptive cluster sampling", Adv. Appl. Stat., 53(1), pp. 13-28 (2018).

6. Ba̧k, T. "An extension of Horvitz-Thompson estimator used in adaptive cluster sampling to continuous universe", Comm. Statist. Theory Methods, 46(19), pp. 9777-9786 (2017).

7. Younis, F. and Shabbir, J. "Estimators for population mean in adaptive cluster sampling", Thail. Stat., 15(2), pp. 105-110 (2017).

8. Younis, F. and Shabbir, J. "A class of Hartleyross-type estimators for population mean in adaptive and stratified adaptive cluster sampling", Iran. J. Sci. Technol. Trans. Sci., 43, pp. 1619-1627 (2019). https://doi.org/10.1007/s40995-018-0552-6

9. Younis, F. and Shabbir, J. "Generalized ratio-type and ratio-exponential-type estimators for population mean under modified Horvitz-Thompson estimator in adaptive cluster sampling", J. Stat. Comput. Simul., 89(8), pp. 1505-1515 (2019).

10. Younis, F. and Shabbir, J. "Estimation of general parameter in adaptive cluster sampling using two auxiliary variables", J. Natn. Sci. Foundation Sri Lanka, 47(1), pp. 89-103 (2019).
11. Haq, A., Khan, M., and Hussain, Z. "A new estimator of finite population mean based on the dual use of the auxiliary information", Comm. Statist. Theory Methods, 46, pp. 4425-4436 (2017).

12. Shabbir, J. "Efficient utilization of two auxiliary variables in stratified double sampling", Comm. Statist. Theory Methods, 47(1), pp. 92-101 (2017).

13. Shabbir, J. and Gupta, S. "On generalized exponential chain ratio estimators under stratified two-phase random sampling", Comm. Statist. Theory Methods, 46, pp. 2910-2920 (2017).

14. Gupta, S. and Shabbir, J. "On the use of transformed auxiliary variables in estimating population mean by using two auxiliary variables", J. Stat. Plan. Inference, 137(5), pp. 1606-1611 (2007).

15. Singh, H.P., Upadhyaya, L.N., and Tailor, R. "Ratiocum-product type exponential estimator", Statistica, 69(4), pp. 299-310 (2009).

16. Choudhury, S. and Singh, B.K. "A class of chain ratioproduct type estimators with two auxiliary variables under double sampling scheme", J. Korean Stat. Soc., 41(2), pp. 247-256 (2012).

17. Hamad, N., Hanif, M., and Haider, N. "A regression type estimator with two auxiliary variables for twophase sampling", Open Journal of Statistics, 3, pp. 74-78 (2013).

18. Chutiman, N. "Adaptive cluster sampling using auxiliary variable", Journal of Mathematics and Statistics, 9(3), pp. 249-255 (2013).

19. Yadav, S.K., Misra, S., Mishra, S.S., and Chutiman, N. "Improved ratio estimators of population mean in adaptive cluster sampling", J. Stat. Appl. Prob. Lett, 3(1), pp. 1-6 (2016).

20. Qureshi, M.N., Kadilar, C., Noor U1 Amin, M., and Hanif, M. "Rare and clustered population estimation using the adaptive cluster sampling with some robust measures", J. Stat. Comput. Simul., 88(14), pp. 27612774 (2018).

21. Vishwakarma, G.K. and Gangele, R.K. "A class of chain ratio-type exponential estimators in double sampling using two auxiliary variates", Appl. Math. Comput., 227, pp. 171-175 (2014).

22. Singh, G.N. and Khalid, M. "Exponential chain dual to ratio and regression type estimators of population mean in two phase sampling", Statistica, 75(4), pp. 379-389 (2015).

23. Khan, M. and Al-Hossain, A.Y. "A note on a difference-type estimator for population mean under two-phase sampling design", SpringerPlus, 5(1), 723 (2016). DOI: $10.1186 / \mathrm{s} 40064-016-2368-1$

24. Khan, M. "A ratio chain-type exponential estimator for finite population mean using double sampling", SpringerPlus, 5, p. 86 (2016). DOI: 10.1186/s40064016-1717-4

25. Singh, H.P., Pal, S.K., and Solanki, R.S. "A new class of estimators of finite population mean in sample 
surveys", Comm. Statist. Theory Methods, 46, pp. 2630-2637 (2017).

26. Shabbir, J. and Gupta, S. "Estimation of finite population mean in simple and stratified random sampling using two auxiliary variables", Comm. Statist. Theory Methods, 46(20), pp. 10135-10148 (2017).

27. Muneer, S., Shabbir, J., and Khalil, A. "Estimation of finite population mean in simple random sampling and stratified random sampling using two auxiliary variables", Comm. Statist. Theory Methods, 46(5), pp. 2181-2192 (2017).

28. Singh, R., Chauhan, P., Sawan, N., and Smarandache, F. "Improved exponential estimator for population variance using two auxiliary variables", Octogon Math. Mag., 17(2), pp. 667-674 (2009).

29. Singh, H.P. and Solanki, R.S. "A new procedure for variance estimation in simple random sampling using auxiliary information", Statist. Papers, 54(2), pp. 479497 (2013).

30. Yunusa, O. and Kadilar, C. "A study on the chain ratio-type estimator of finite population variance", Journal of Probability and Statistics, 2014, Article ID 723982, 5 pages (2014).

https://doi.org/10.1155/2014/723982

31. Amin, M.N.U., Yasmeen, U., and Hanif, M. "Generalized variance estimators in adaptive cluster sampling using single auxiliary variable", J. Stat. Manag. Syst., 21(3), pp. 401-415 (2018).
32. Smith, D.R., Conroy, M.J., and Brakhage, D.H. "Efficiency of adaptive cluster sampling for estimating density of wintering waterfowl", Biometrics, 51(2), pp. $777-788$ (1995).

33. Dryver A.L. and Chao, C.-T. "Ratio estimators in adaptive cluster sampling", Environmetrics, 18(6), pp. 607-620 (2007).

34. Chao, C.-T., Dryver, A.L., and Chiang, T.C. "Leveraging the rao-blackwell theorem to improve ratio estimators in adaptive cluster sampling", Environ. Ecol. Stat., 18(3), pp. 543-568 (2011).

35. Zamanzade, E. and Vock, M. "Variance estimation in ranked set sampling using a concomitant variable", Statist. Probab. Lett., 105, pp. 1-5 (2015).

\section{Biographies}

Faryal Younis obtained her $\mathrm{PhD}$ degree in Statistics from Quaid-i-Azam University, Pakistan in March 2019. Her areas of interest include sampling theory and Bayesian analysis.

Javid Shabbir is a Professor of Statistics at Quaidi-Azam University, Pakistan. He has 200 research publications in reputable journals. His areas of interest include survey sampling, nonresponse, and randomized response. 